\title{
High-pressure synthesis and physical properties of new iron(nickel)-based superconductors
}

P. M. Shirage ${ }^{1}$, K. Miyazawa ${ }^{1,3}$, M. Ishikado ${ }^{1,2}$, K. Kihou $^{1}$, C. H. Lee ${ }^{1,4}$, N. Takeshita ${ }^{1,4}$, H. Matsuhata ${ }^{1,4}$, R. Kumai ${ }^{1,4}$, Y. Tomioka ${ }^{1,4}$, T. Ito ${ }^{1,4}$, H. Kito ${ }^{1,4}$, H. Eisaki ${ }^{1,4}$,

S. Shamoto ${ }^{2,4}$, A. Iyo ${ }^{1,3,4}$

${ }^{1}$ National Institute of Advanced Industrial Science and Technology (AIST), 1-1-1

Central 2, Umezono, Tsukuba, Ibaraki, 305-8568, Japan.

${ }^{2}$ Quantum Beam Science Directorate, Japan Atomic Energy Agency, Tokai, Naka, Ibaraki, 319-1195, Japan.

${ }^{3}$ Department of Applied Electronics, Tokyo University of Science, 2641 Yamazaki, Noda, Chiba 278-3510, Japan.

${ }^{4}$ JST, Transformative Research-Project on Iron Pnictides (TRIP), 5, Sanbancho, Chiyoda, Tokyo 102-0075, Japan.

E_mail : paras-shirage@aist.go.jp, h-eisaki@aist.go.jp, iyo-akira@aist.go.jp 
Abstract

1. Introduction

2. Sample preparation by HP technique

2.1 High Pressure Synthesis technique

2.2 Synthesis of O-deficient $\mathrm{LnFeAsO}_{1-y}$

2.2.1 Polycrystalline samples

2.2.2 Single crystals

2.3 Synthesis of $A M_{2} X_{2}$ samples

3. Structural and physical properties of $2 n \mathrm{FeAsO}_{1-\mathrm{y}}$

3.1 The synthesis of $N d F e A S O_{1-y}$ samples with controlled oxygen deficiency

3.2 Effect of synthesis pressure on $\mathrm{LnFeAs}_{1-y}$ with heavy Ln's

3.3 Universal superconducting transition temperature of $\mathrm{LnFeAsO}_{1-y}$

3.4 Physical Properties of $\mathrm{LnFeAs} \mathrm{O}_{1-y}$ under high pressure

3.4.1 Electrical resistivity measurement under pressure

3.4.2 The crystal structure under pressure

3.5 Relationship between $T_{c}$ and crystal structure

3.6 Properties of $\mathrm{PrFeAs}_{1-y}$ single crystals

4. The synthesis and physical properties $A M_{2} X_{2}$ samples

$4.1(\mathrm{Ca}, \mathrm{Na}) \mathrm{Fe}_{2} \mathrm{As}_{2}$

$4.2(\mathrm{Ba}, \mathrm{K}) \mathrm{Fe}_{2} \mathrm{As}_{2}$

4.3 $\mathrm{BaNi}_{2} \mathrm{P}_{2}$ single crystal

5. Summary

References 


\section{Abstract.}

We have utilized a high-pressure (HP) technique to synthesize a series of newly-discovered iron (nickel) - based superconductors. For the LnFeAsO-based superconductors ( $L n=$ lanthanide), we show that the introduction of oxygen (O)-deficiency in the $L n \mathrm{O}$ layers, which is achievable only through HP process, is an effective way to dope electron carriers into the system, which results in yielding the superconducting transition temperature $\left(T_{c}\right)$ comparable with those for F-substituted counterpart. The effect of O-deficiency, variation of $L n$ ions, and the external pressure on $T_{c}$ are examined. All the experimental data indicate strong correlation between the crystal structure and the superconductivity of the oxypnictide superconductors. Upper critical field measurement of $\mathrm{PrFeAsO}_{1-y}$ shows the superconducting anisotropy of 5, which is smaller than cuprates. We also demonstrate that HP technique is applicable for the so-called ' 122 ' systems, by showing the results on polycrystalline $(\mathrm{Ca}, \mathrm{Na}) \mathrm{Fe}_{2} \mathrm{As}_{2},(\mathrm{Ba}, \mathrm{K}) \mathrm{Fe}_{2} \mathrm{As}_{2}$, as well as single crystal $\mathrm{BaNi}_{2} \mathrm{P}_{2}$ samples.

PACS numbers: 74.70.-b, 74.62.Bf, 74.62.Dh, 74.25.-q, 74.62.Fj.

Keywords: Iron (Nickel)-based, Oxypnictide/pnictide superconductors; High pressure synthesis; X-ray diffraction, Transport properties 


\section{Introduction}

The discovery of $\mathrm{LaFeAsO}_{1-x} \mathrm{~F}_{x}$ with $T_{c}=26 \mathrm{~K}$ by Kamihara et al. triggered a tremendous amount of research activities in the field of high-transition temperature (high- $T_{c}$ ) superconductivity [1-13] and the new generation of HTSC is evolved. To date, $T_{c}$ exceeds $50 \mathrm{~K}$, which makes the iron-pnictide superoconductors highest $T_{c}$ materials besides of cuprates. It is also demonstrated that the new superconductors are rich in the material variation, which include other transition metal (TM)'s (Ni, etc.), pnicogen elements (P, As etc.), or even chalcogens ( $\mathrm{S}, \mathrm{Se}, \mathrm{Te}$ etc)[14-16].

To explore new materials, high-pressure (HP) synthesis technique, a method to synthesize materials under high temperature (up to $1500^{\circ} \mathrm{C}$ ) and high pressure (up to several giga-Pascals), is established as one of the most powerful tools. The usefulness has been eloquently demonstrated throughout the history of cuprate superconductors, where a lot of new high- $T_{c}$ superconductors have been discovered and multi-layer superconductors are stabilized and utilized as a $T_{c}$ sprouting technique [17-21].

In many senses, HP synthesis is more advantageous in comparison to the conventional solid state reaction, such as the controlled modification of the phase diagram, enhanced reactivity, and prevention of the evaporation, etc. These advantages turned out to be extremely helpful in synthesizing pnictide superconductors, some of 
which are new, and some are synthesized in single crystalline form.

The first outcome of HP technique was reported by Z. A. Ren et al.[22 ], who succeeded in synthesizing $\operatorname{PrFeAsO}_{1-x} \mathrm{~F}_{x}$ with $T_{c}$ of $52 \mathrm{~K}$. Shortly after that, the same authors reported the $55 \mathrm{~K}$ superconductivity in $\mathrm{SmFeAsO}_{1-x} \mathrm{~F}_{x}[23]$, which marks the highest- $T_{c}$ record among all the Fe-based superconductors discovered so far.

In the middle of April, a new variation of the $L n F e A s O$-based superconductors was discovered by Z. A. Ren et al.[9] and H. Kito et al.[2], independently. The material they have synthesized is oxygen $(\mathrm{O})$-deficient $L n \mathrm{Fe} \mathrm{AsO}_{1-\delta}$, in which O-deficiency plays a role as a charge supplier. In contrast to the original F-substituted system, the O-deficient samples can be synthesized only by adopting HP technique, although their physical properties including their $T_{c}$ 's are quite alike with each other.

From the exploration of the new physics point of view, availability of well-characterized samples with controlled chemistry and single crystal is critically important for elucidating fundamental features. It turned out that HP technique is quite suitable for the purpose. Indeed, as will be shown later, HP technique allowed us to prepare a series of $L n \mathrm{FeAsO}_{1-\mathrm{y}}$ polycrystalline samples with the doping range spanning from undoped non-superconducting to optimal-doped highest- $T_{c}$ regions, and covering variety of $L n$ elements from $L n=\mathrm{La}$ to Dy, which is beyond the accomplishment of 
conventional solid reaction method. Using these samples, we have carried out systematic crystal structural and physical property measurements including under extremely high pressures, and found out the strong correlation between the crystal structures and the superconductivity in this system. Furthermore, single crystal samples of $\mathrm{BaNi}_{2} \mathrm{P}_{2}$ grown by HP technique provided us with the opportunity to evaluate the anisotropic superconducting properties, which will be also covered in this article.

In this paper, we summarize our current status of HP sample synthesis and the experimental results obtained using these samples. In section 2, we describe the sample synthesis of $L n \mathrm{FeAsO}_{1-\mathrm{y}},(\mathrm{Ba}, \mathrm{K}) \mathrm{Fe}_{2} \mathrm{As}_{2},(\mathrm{Ca}, \mathrm{Na}) \mathrm{Fe}_{2} \mathrm{As}_{2}$, and $\mathrm{BaNi}_{2} \mathrm{P}_{2}$ in detail, together with the general aspects of HP technique. Section 3 deals with the physical properties of $L n \mathrm{FeAsO}_{1-\mathrm{y}}$, in which we stress the intimate correlation between the crystal structure and superconductivity. Physical properties of $\mathrm{PrFeAsO}_{1-y}$ single crystals are also included in section 3. Section 4 is devoted to the "122" phase.

\section{Sample preparation by HP technique}

\subsection{High Pressure synthesis technique}


For synthesizing any material, it is critically important to sort out adequate synthesis process, such as the choice of starting materials, reaction temperature, atmosphere, etc. HP synthesis provides us with a number of advantages over conventional solid state reaction.

1. Pressure modifies the phase diagrams of matters towards the direction which favors smaller volume. This results in extending the solubility limit of constituent elements, or forming new compounds with their volume much smaller (high density) than those obtained under ambient conditions which are unstable.

2. HP synthesis is a high-throughput process, effective for hunting new materials. Owing to the enhanced reactivity under HP, one can considerably shorten the periods required for the sample synthesis process, even by a factor of 10 . This is extremely useful if one try to develop new materials.

3. By adopting HP technique, one can easily prevent the evaporation of toxic and/or volatile elements, such as As, $\mathrm{K}$, and $\mathrm{F}$, and its reaction with the sample containers which are typically made of quartz ampoules, sometimes resulting in the explosion during the ambient 
pressure synthesis.

4. HP synthesis is crucial technique for high quality single crystal growth since high temperature is achievable beyond the melting of the quartz ampoules, although the sample space is limited where the temperature gradient is large due to the thermal leak from surrounding anvil.

Disadvantage of the HP technique should be noted. First, small sample volume limits the amount of the charged material, putting the limitation of the grown samples. Second, thermal leak from the surrounding anvils may cause temperature gradient within sample space.

In our study, HP synthesis is carried out using wedge-type cubic-anvil high pressure apparatus (TRY engineering Co.Ltd., Kawasaki, Tokyo, Japan) and photo is shown in Fig. 1(a). The starting materials are encapsulated into closed containers made up of boron nitride $(\mathrm{BN})$ sleeve, and then embedded into solid media (pyrophyllite $\left.\left[\mathrm{Al}_{2} \mathrm{Si}_{4} \mathrm{O}_{10}(\mathrm{OH})_{2}\right]\right)$ which transmit pressures typically up to several-GPa to the samples. The samples are heated up in-situ using electrical heater which surrounds the sample container (BN sleeve), also embedded into pressure media. The configuration of the sample cell is shown in Fig. 1(b). 


\subsection{Synthesis of O-deficient $\mathrm{LnFeAsO}_{1-y}$}

\subsubsection{Polycrystalline samples}

A typical flowchart of HP synthesis procedure is represented in Fig. 2. The starting materials are: grained $L n$ metal soaked in oil (rare metallic, Soekawa Chem. Furuuchi Chem. 99.9\%, 20mesh), As-chips with particle diameter 1-4mm (High Quality Chem, $6 \mathrm{~N}$ ), Fe powder (Furuuchi Chem, 99.9\%, 100mesh), and $\alpha-\mathrm{Fe}_{2} \mathrm{O}_{3}$ powder (rare metallic, 99.9\%).

Before HP synthesis, we have synthesized $L n$ As precursors by reacting $L n$ and As. Compared to $L n$ ingots, reacted $L n A s$ is relatively brittle and thus helpful for mixing starting elements homogeneously which is responsible for the purity of the phase.

Ln and As are weighed with a molar ratio 1:1 and then sealed into a quartz ampoule. The ampoule is heated up to $500^{\circ} \mathrm{C}$ at a rate $50^{\circ} \mathrm{C} / \mathrm{h}$ and held for $15 \mathrm{~h}$, and then elevated to $850^{\circ} \mathrm{C}-950^{\circ} \mathrm{C}(\mathrm{La}, \mathrm{Ce}, \mathrm{Pr}, \mathrm{Nd})$ or $1000^{\circ} \mathrm{C}(\mathrm{Sm}, \mathrm{Gd}$, etc $)$ at a rate $50^{\circ} \mathrm{C} / \mathrm{h}$, and held for $10 \mathrm{~h}$, followed by a furnace cooling. Since the resultant $L n A s$ is susceptible to oxidation and releases toxic arsine $\left(\mathrm{AsH}_{3}\right)$ gas when reacted with moisture, precaution in handling is required. It is strongly recommended that all the process is carried out 
within a dry, oxygen-free glove box.

$L n A s, \mathrm{Fe}, \mathrm{Fe}_{2} \mathrm{O}_{3}$ powders are weighed with their molar ratio $L n: \mathrm{Fe}: \mathrm{As}: \mathrm{O}=1: 1: 1: 1-\mathrm{y}$. The amount of nominal oxygen content was controlled by changing the ratio of Fe and $\mathrm{Fe}_{2} \mathrm{O}_{3}$. As described later, we have obtained the optimal superconductivity at the nominal oxygen content $1-y=0.6-0.7$. However, subsequent neutron diffraction studies on the same samples yields the real oxygen content larger than the nominal one. The discrepancy arises from oxidation either of the starting materials ( $\operatorname{LnAs}, \mathrm{Fe})$ or during HP synthesis.

The powders are mixed, and pressed into pellet. The pellet is encapsulated into a BN sleeve and HP treated under a several GPa pressure. As described later, the optimal pressure depends on $L n$. For lighter $L n$ 's $(L n=\mathrm{La}, \mathrm{Ce}, \mathrm{Pr}, \mathrm{Nd}), 2.0 \mathrm{GPa}$ is enough. On the other hand, higher pressures, typically up to $5 \mathrm{GPa}$ is required for synthesizing samples containing heavier $L n$ 's (Sm, Gd, Tb, Dy). The samples are heated up at $1050-1150{ }^{\circ} \mathrm{C}$ depending on $L n$ for 2 hours.

To compare the effect of O-deficiency and F-substitution, as well as the dependence of synthesis method, we also synthesized the F-substituted $L n F e A s O(L n=\mathrm{La}, \mathrm{Nd})$ by conventional solid-state reaction method. We have employed $\mathrm{FeF}_{2}$ as a source of $\mathrm{F}$. The detail of sample synthesis is described in Ref.[24]. 


\subsubsection{Single crystals}

In this study, we have tried to grow single crystals of $\mathrm{PrFeAsO}_{1-\mathrm{y}}$ by self-flux method, using As and FeAs as flux. Powders of PrAs, Fe, $\mathrm{Fe}_{2} \mathrm{O}_{3}$ and As grain were used as the starting materials. The starting materials with their nominal compositions of $\mathrm{PrFeAsO}_{0.7}$ were mixed together with excess flux and then ground by an agate mortar in a glove box filled with dry nitrogen gas. The samples were pressed into pellet.

We have used the belt-type anvil type HP apparatus with sample size as large as $8 \mathrm{~mm} \phi^{*} 9 \mathrm{~mm}$ (2grams). The samples were encapsulated in BN sleeves, and then heat-treated under a pressure of about $2 \mathrm{GPa}$ at $1300-1400^{\circ} \mathrm{C}$ for $2 \mathrm{hrs}$.

\subsection{Synthesis of $A M_{2} X_{2}$ samples}

Synthesis of the $A M_{2} X_{2}(\mathrm{~A}=\mathrm{Ba}, \mathrm{Ca}, M=\mathrm{Fe}, \mathrm{Ni} . X=\mathrm{P}, \mathrm{As})$ series is known to be easier in comparison with the $\mathrm{LnFeAsO}$ series and studies on single crystal samples are mostly carried out on these class of materials. Here the main role of HP synthesis is to prevent the evaporation of volatile elements. (For example, the vapor pressure of phosphor (P) exceeds $10 \mathrm{~atm}$ at $500^{\circ} \mathrm{C}$.) Stoichiometric amount of starting materials, $M(=\mathrm{Fe}, \mathrm{Ni}), X$ 
$(=\mathrm{P}, \mathrm{As})$ and $\mathrm{A}(=\mathrm{Ca}$ or $\mathrm{Ba})$ (Furuuchi Chemical Corporation, flake form, $5-20 \mathrm{~mm}$, $99.9 \%$ ) are put into BN sleeves. Single crystals with the size up to $1 \mathrm{~mm}^{2} * 10 \mu \mathrm{m}$ can be obtained by heating the elements for $1-2$ hours at about $1050^{\circ} \mathrm{C}-1200{ }^{\circ} \mathrm{C}$ under 1GPa. Same procedure can be applied for synthesizing polycrystalline sample. In this case lower temperature around $850^{\circ} \mathrm{C}-950^{\circ} \mathrm{C}$ is preferred. While single crystals of $A \mathrm{Fe}_{2} \mathrm{As}_{2}$ can be also obtained by conventional flux methods [25-27], additional flux, such as FeAs or $\mathrm{Sn}$, as well as longer reaction time, is required. In the present case, we have not used any flux, which possibly helps in synthesizing samples free from flux contamination within short time.

\section{Structural and physical properties of $\operatorname{LnFeAsO} \mathrm{O}_{1-y}$}

\subsection{Synthesis of $\mathrm{NdFeAs} \mathrm{O}_{1-y}$ samples with controlled oxygen deficiency}

The superconductivity in $L n F e A s O$ system can be simply induced by oxygen deficiency, rather than F-substitution. This makes $L n F e A s O$ system easier in processing and obtaining pure sample. In Fig.3(a), we show the powder x-ray diffraction (XRD) patterns for $\mathrm{NdFe} \mathrm{AsO}_{1-\mathrm{y}}$ samples synthesized under $2 \mathrm{GPa}$ and at $1150^{\circ} \mathrm{C}$. Nearly all the 
peaks can be indexed on the basis of the tetragonal $\mathrm{ZrCuSiAs}$ type crystal structure with P4/nmm symmetry, as expected for $L n F e A s O$ and its oxygen deficient form, ensuring the good sample quality of the HP samples. In Fig.3 (b), we represent the magnified 212 peak from Fig. 3(a), which distinctly shifts to higher angle (decrease in lattice constants) with increasing nominal oxygen deficiency $(y)$.

Determination of the real $\mathrm{O}$ content $y$ is carried out though the Rietvelt analysis of the powder neutron diffraction data. The detail of the neutron experiments and analysis is described in Ref. [28]. Plotted in Fig. 4 is the $y$ value against the $a$-axis lattice parameters for $\mathrm{NdFeAsO}_{1-y}$, in which a linear relationship between the two parameters is depicted. The relation between oxygen content and $a$-lattice parameter can be represented by formula, $1-y=6.637 a-25.373$.

Shrinkage of the lattice constant with $y$ is reasonable, since the oxygen deficiency alters the charge distribution between $\mathrm{NdO}$ layers and FeAs layers. Stoichiometric $\mathrm{NdFeAsO}$ is composed of the alternating stack of $(\mathrm{NdO})^{+1}$ and $(\mathrm{FeAs})^{-1}$ layers. Upon removal of $\mathrm{O}$, charge distribution changes into $\left(\mathrm{NdO}_{1-\delta}\right)^{+1+2 \delta}$ and $(\mathrm{FeAs})^{1-2 \delta}$, which causes the displacement of these layers closer to each other, thus resulting in the shrinkage of $c$-axis. This also explains why HP is effective in introducing O-deficiency, since HP tend to stabilize materials with smaller unit cell volumes. 
In Fig. 5, we plot the magnetic susceptibility data for $\mathrm{NdFeAsO}_{1-y} . T_{c}$ of the samples increases monotonously with $y$. Minimal doped sample $1-y=0.96$ does not show superconductivity above $\mathrm{T}=5 \mathrm{~K}$, whereas the other samples $(1-y=0.92,0.88$, and 0.84$)$ shows superconductivity with onset $T_{c}=32 \mathrm{~K}, 48 \mathrm{~K}$, and $51 \mathrm{~K}$ respectively.

Figure 6 shows a typical temperature-dependence of the resistivity. For the sample $1-y=0.96$ which does not show superconductivity, around $140 \mathrm{~K}$ an anomaly driven by a spin density wave (SDW) instability and/or a structural phase transition which is commonly observed among the parent compounds of iron-arsenide, is observed. For the sample $1-y=0.80$ with the highest $T_{c}$ (onset $54 \mathrm{~K}$ ), the resistivity varies almost linearly (or slightly curved into a s-shape). It is interesting that the optimally-doped cupper-oxide superconductors show similar behavior. Oxygen doping dependence with $L n=\mathrm{Nd}$ shows the same $T_{c}$ as F-doped samples, representing the electron doping mechanism is same and apparently same phase diagram from non-superconducting to superconducting transition.

Figure 7 shows the electronic phase diagram obtained by plotting the $a$-parameter and $T_{\mathrm{c}}$ for the $\mathrm{NdFeAsO}_{1-y}$ samples. $T_{\mathrm{c}}$ is determined from the onset of transition in magnetic susceptibility. The upper scale shows the oxygen content 1-y estimated from Fig. 4. Boundary between superconducting state and 
non-superconducting one is very clear. When the $a$-parameter is reduced from that of non-superconducting sample, the $T_{\mathrm{c}}$ abruptly increases at about $a=3.964 \AA(1-y=0.94)$ and saturates about $54 \mathrm{~K}$ at $a=3.94 \sim 3.95 \AA$. Superconductivity appears when $a$ is shortened by about 0.01 angstrom compared to the non-SC parent compound, which occurs when $y=0.06$. Z.A. Ren et al. also shows the phase diagram of $\mathrm{NdFeAsO}_{1-\mathrm{d}}$, where the $T_{\mathrm{c}}$ decreases for further shrink of the $a$-parameter $(\sim 3.92 \AA)[9]$. We have not obtained such samples having lower $T_{\mathrm{c}}$ and short $a$-parameters.

\subsection{Effect of synthesis pressure on $\mathrm{LnFeAsO}_{1-y}$ with heavy Ln's}

For the heavier $L n$ 's $(L n=\mathrm{Gd}, \mathrm{Tb}$ and $\mathrm{Dy})$, the sample quality and their superconducting properties strongly depends on the synthesis conditions like temperature and pressure etc. For example, $T_{c}$ of ambient pressure (AP)-synthesized $\mathrm{GdFeAsO}_{1-\mathrm{x}} \mathrm{F}_{\mathrm{x}}$ sample is $30 \mathrm{~K}$, much lower than those for $L n=\mathrm{Nd}$ or $\mathrm{Sm}$ synthesized under the same conditions[29]. For $L n=\mathrm{Tb}$ and Dy, HP technique is the only way to synthesize superconducting samples. Furthermore, to our knowledge, there is no report on the successful synthesis of single-phase Dy- and Tb- based superconductors with their $T_{c}$ 's exceeding $50 \mathrm{~K}$, which lead one to suspect that $T_{c}$ drops down with increasing Ln atomic number. 
The unit cell volume of $L n F e A s O$ is expected to decrease with increasing the $L n$ atomic number, following the shrinkage of $L n$ ionic radius (lanthanide contraction). It is thus expected that HP technique is effective for the formation of the $L_{n F e A s O}$ (and $L n F e A s \mathrm{O}_{1-\mathrm{x}} \mathrm{F}_{\mathrm{x}}$ ) phase with heavier $L n$ ions. This proposed hypothesis turned out to work excellently. To demonstrate our hypothesis, we show in Fig. 8 the powder XRD patterns for the samples with a nominal composition of $\mathrm{DyFeAsO}_{0.7}$. Here the synthesis pressure is varied from 2.0 (top) to $5.5 \mathrm{GPa}$ (bottom). Impurity peaks, mainly due to DyAs, are present in the samples synthesized at lower pressures, up to $3.5 \mathrm{GPa}$. Note that the pressure range $2-3.5 \mathrm{GPa}$ is suitable for synthesizing single phase, superconducting $\mathrm{NdFeAsO}_{1-y}$. The impurity phase decreases when pressure is increased, demonstrating that adopting $\mathrm{HP}$ is effective to stabilize the $\mathrm{DyFeAsO}_{0.7}$ phase. Indeed, at the same time, the diffraction peaks systematically shift toward the higher diffraction angle (shown in Fig.8(b)), indicating that the lattice parameters shrink with increasing the pressure, consistent with the aforementioned expectation. Corresponding to the shrinkage of the lattice parameters with pressure, $T_{c}$ also increase as shown in the temperature dependence of the magnetic susceptibility (Fig. 9). This result indicates strong relation between pressure of synthesis, $a$-lattice parameter, and $T_{c}$.

This experimental result suggests that the effective role of HP is to stabilize the 
O-deficient crystal structure which possesses smaller unit cell volume. If this is the case, one expects the increment of $T_{c}$ with decreasing lattice constants due to the introduction of O-deficiency, like $\mathrm{NdFeAsO}_{1-y}$. Fig.10 indeed confirms the expectation, in which the clear relationship between $T_{\mathrm{c}}$ and the $a$-axis lattice parameter is visible for $L n \mathrm{FeAsO}_{0.7}$ with $L n=\mathrm{Sm}, \mathrm{Gd}, \mathrm{Tb}$ and Dy which are synthesized under various pressures ranging from 2.0 to $5.5 \mathrm{GPa}$. Increase of the synthesis pressure results in the decrease of $a$, along with the increase of $T_{\mathrm{c}}$, especially for $L n=\mathrm{Tb}$ and Dy. For all $L n$ 's, the highest $T_{c}$ 's which exceed $50 \mathrm{~K}$ are recorded for the samples synthesized under high pressures, typically $5.0-5.5 \mathrm{GPa}$.

\subsection{Universal superconducting transition temperature of $\mathrm{LnFeAsO}_{1-y}$}

Once the synthesis condition is intimately optimized, one can compare $T_{c}$ 's of $L n \mathrm{FeAsO}_{1-\mathrm{y}}$ with different $L n$ 's. Fig. 11 (a) to (h) show the $T$ - dependence of the magnetic susceptibility for $L n \mathrm{FeAsO}_{1-\mathrm{y}}(\mathrm{Ln}=\mathrm{La}, \mathrm{Ce}, \mathrm{Pr}, \mathrm{Nd}, \mathrm{Sm}, \mathrm{Gd}, \mathrm{Tb}$ and Dy). Here we show the data of the highest $T_{c}$ samples for each $L n$ series, which means that the data presented in Fig. 11 provide the lower boundaries of the attainable $T_{c}$ 's for each series. The sharp drops of the magnetic susceptibility, corresponding to the onset of superconductivity (indicated by arrows in Fig. 11), are observed. $T_{\mathrm{c}}$ increases from 
$L n=\mathrm{La}$ to $\mathrm{Nd}$ and apparently exceeds $50 \mathrm{~K}$ for $L n=\mathrm{Nd}, \mathrm{Sm}, \mathrm{Gd}, \mathrm{Tb}$ and $\mathrm{Dy}$.

Fig. 12 (a) to (h) shows $T$ - dependence of the resistivity for $L n=\mathrm{La}, \mathrm{Ce}, \mathrm{Pr}, \mathrm{Nd}, \mathrm{Sm}, \mathrm{Gd}$, $\mathrm{Tb}$ and Dy, respectively. All the samples show metallic $(d \rho / d T>0)$ behaviour in all $T$-range. As shown in the insets, sharp superconducting transitions are observed. Again, one can see the same tendency as the susceptibility. $T_{c}$ increases from $L n=\mathrm{La}$ to $\mathrm{Nd}$ and exceeds $50 \mathrm{~K}$ for $L n=\mathrm{Nd}, \mathrm{Sm}, \mathrm{Gd}, \mathrm{Tb}$ and $\mathrm{Dy}$.

The $L n$-dependence of the highest $T_{\mathrm{c}}$ is summarized in Fig. 13, which reveals the systematic tendency against the $a$-axis lattice parameter. $T_{\mathrm{c}}$ increases from $28 \mathrm{~K}$ $(L n=\mathrm{La})$ to $53 \mathrm{~K}(L n=\mathrm{Nd})$, almost linearly to the $a$-axis parameter. Once $T_{\mathrm{c}}$ reaches $53 \mathrm{~K}$, it stays almost constant from $L n=\mathrm{Nd}$ to Dy. As mentioned, at this moment one cannot rule out the possibility for further increase of $T_{c}$ by finer tuning of the synthesis conditions. However, as far as the observed tendency is concerned, the current results appear to suggest the existence of an upper limit of $T_{c}$ for the $L n F e A s O_{1-y}$ system, which is located around $53 \mathrm{~K}$.

\subsection{Physical properties of $\mathrm{LnFeAsO}_{1-y}$ under high pressure}

\subsubsection{Electrical resistivity measurements under pressure}


Samples synthesized under HP are useful for physical properties measurements, thanks to higher quality and higher density. As one of the examples, here we summarize the results on high-pressure transport properties measurements on $\mathrm{NdFeAsO}_{1-\mathrm{y}}[30,31]$, with $T_{c}=0 \mathrm{~K}(1-y($ nominal $)=0.85), 40 \mathrm{~K}(1-y($ nominal $)=0.8)$, and $54 \mathrm{~K}(1-y($ nominal $)=0.6)$.

We employed cubic anvil type apparatus[32] for generating nearly hydrostatic high pressure up to $18 \mathrm{GPa}$. During cooling and warming processes, the applying load was kept at the constant value against the thermal expansion of the cryostat, therefore the pressure values are enough reliable at all temperatures. Since the cubic anvil apparatus pressurizes the sample from isotropic six directions, we can anticipate nearly hydrostatic high pressure inside. Additionally, to be careful about inhomogeneity of pressure generating inside the sample space, we used mixture of Fluorinert (FC70 : FC77 = 1: 1) or Daphne 7474 oil[33], as liquid pressure transmitting medium to moderate the anisotropy.

Fig. 14 represents the pressure dependence of $T_{c}$ (defined from the onset of resistivity drop) of the $\mathrm{NdFeAsO}_{0.6}$ and $\mathrm{NdFeAsO}_{0.8}$ samples, while Inset of Fig. 15 shows the temperature dependence of the resistivity of $\mathrm{NdFeAsO}_{0.8}$ under different pressures. $T_{\mathrm{c}}$ 's of both samples monotonically decrease with increasing pressure.

Earlier reports on the La-system by Takahashi et al. indicate the increase in $T_{c}$ 
with pressure, which has been ascribed to the pressure-induced carrier doping [34]. If this is the case, one should expect the increase of $T_{c}$ in the possibly under-doped $\mathrm{NdFeAsO}_{0.8}$ sample towards 54K. However, present results contradict with the expectation. This indicates the breakdown of simple carrier-doping picture, as well as suggesting the different role of crystal structure on the superconductivity between the La- and Nd-based systems, which likely results in different $T_{c}$ even under ambient pressure.

Fig. 15 shows the temperature dependence of the resistivity of $\mathrm{NdFeAsO}_{0.85}$ sample under different pressures. The resistivity anomaly is visible around $150 \mathrm{~K}$ under ambient pressure, which is suppressed upon pressure in similarity with the formation of O-deficiency. Concomitantly, the drop in the resistivity shows up at low temperatures above $8 \mathrm{GPa}$, with the onset temperature increases with pressure. Eventually, at around $16 \mathrm{GPa}$, sudden fall in the resistivity appears around $20 \mathrm{~K}$, which might be the signature of superconductivity. However, we always encountered finite resistivity even at the highest pressure of $16 \mathrm{GPa}$. Since the resistivity depends on the applied current, it may be superconducting transition. Then we have to suspect the origin of the behavior. The data clearly show that pressure has the effect on suppressing the resistivity anomaly of the parent compound, which is likely to come from SDW order. In addition, it is likely 
that the resistivity anomaly competes with superconductivity. Three possibilities can be considered:

(i) By applying pressure, the SDW transition is suppressed and superconductivity is induced. However, since the doping content is too small (compared to chemical doping by O-deficiency), signature of superconductivity is weak.

(ii) An impurity phase which shows pressure-induced superconductivity might exist.

(iii) By suppressing SDW, FeAs planes intrinsically show bulk superconductivity even in a non-doped sample. However, the qualities of the samples are not perfect yet.

It is too early to make the firm conclusion. We note that similar phenomenon is also observed in other parent compound, LaFeAsO [35].

Recent observation of pressure-induced superconductivity observed in $\mathrm{AFe}_{2} \mathrm{As}_{2}$ $(\mathrm{A}=\mathrm{Ca}, \mathrm{Sr}, \mathrm{Ba})$, parent compounds of ' 122 ' systems, may support above (ii) and (iii) scenarios [36-40]. However, perfection of the samples is critical to investigate the intrinsic feature. Actually, the absence of the superconductivity in pure single crystalline samples under highly homogeneous pressure have been reported[41, 42]. In the future, further experimental studies, such as specific heat and NMR, etc., are necessary in order 
to sort out the nature of superconductivity under high pressure.

\subsubsection{The crystal structure under pressure}

The primary effect of pressure on a sample is the compression of the crystal lattice, as well as the displacement of the atoms in the unit cell. Theoretical calculations also suggest susceptible change of the electronic structure in response to the displacement of the atoms [43], which may be related to the rapid decrease of $T_{\mathrm{c}}$ under pressure as shown in the former section. In order to determine the crystal structure of $\mathrm{NdFeAsO}_{0.7}$ (nominal composition), we carried out X-ray diffraction measurements under high pressure at $\underline{\mathrm{PF} \text { BL-1A, }}$, and analyzed the data by the Rietveld method [44]. A diamond-anvil cell (DAC) with $0.8 \mathrm{~mm}$ tip diamonds was used for the diffraction measurements under high pressure. Daphne 7373 oil was used for the pressure transmitting medium.

Fig. 16 shows the pressure dependence of the relative lattice constants. Both of

the lattice constants decrease monotonically as a function of the pressure. The compressibility along the $c$-axis direction is much larger than that along the $a$-axis, which would be related to the anisotropy of the chemical bonds. Fig. 17 shows the 
pressure dependence of the crystal structure parameters, which are related to the displacement of the atoms in the unit cell. The change in the bond angle (Fig.17 (b)) is comparatively small, while bond length (Fig.17 (a)) and distance between planes (Fig. 17 (c)) are much shrunk. The large compressibility along the $c$-axis reflects the distance between planes shortened greatly, which is characteristic of this system. Although it is difficult to explain the change of $T_{\mathrm{c}}$ from the change of the crystal structure obtained so far, it is expected that further high pressure experiments on the samples which have different oxygen deficiency or contain other Lanthanides would make clear the relationship between the $T_{\mathrm{c}}$ and the structure.

\subsection{Relationship between $T_{c}$ and crystal structure}

The qualities of the HP synthesized samples are useful for the detailed crystal structural analysis. It is particularly important, given that $T_{\mathrm{c}}$ of iron-based superconductors dramatically changes by changing the crystal structural parameters through the $L n$ substitution and/or the application of high pressure. The observations lead us to examine the crystal structure in detail, by using the powder neutron diffraction technique on $\mathrm{NdFeAsO}_{1-\mathrm{y}}$ samples. We focus our attention on the angle $\alpha$ which characterizes the shape of $\mathrm{FeAs}_{4}$-tetrahedron. In Fig.18, we plot the relationship between $T_{c}$ and $\alpha$ of $L n \mathrm{FeAsO}_{1-y}(L n=\mathrm{La}, \mathrm{Nd})$. Clear correlation between $\alpha$ 
and $T_{c}$ is established. In particular, $T_{\mathrm{c}}$ increases as $\mathrm{FeAs}_{4}$-tetrahedrons approaches regular shape, in which $\alpha$ corresponds to $109.47^{\circ} \alpha v s . T_{\mathrm{c}}$ relationship in various pnictide superconductors are summarized in Fig. 19. Obviously, $T_{\mathrm{c}}$ becomes maximum when $\mathrm{FeAs}_{4}$ tetrahedron form a regular shape, indicating that there is a strong correlation between the crystal structure and the superconductivity.

\subsection{Properties of $\mathrm{PrFeAsO}_{1-y}$ single crystals}

In order to understand the intrinsic physical properties, studies on single crystals are highly demanded. In particular, most of the advanced experimental techniques which play roles in elucidating high- $T_{c}$ mechanism on cuprate superconductivity, such as Angle-Resolved Photoemission Spectroscopy (ARPES), Scanning Tunneling Spectroscopy (STS), optical spectroscopy, etc., require high-quality, sizable single crystal samples. In order to obtain large single crystals with sufficient quality, we have employed the HP technique.

We successfully obtained single crystal of $\mathrm{PrFeAsO}_{0.7}$ when $20 \%$ excess As was added[45]. Fig. 20 represents the plate-like single crystals with maximum size of $\sim 1 \mathrm{~mm}^{2} \times 50 \mu \mathrm{m}$ were embedded in the bulk of pellet. The Laue diffraction confirmed the single crystalline nature of the obtained samples. 
Susceptibility measurements show that $T_{\mathrm{c}}$ of the single crystals is about $44 \mathrm{~K}$, as shown in Fig. 21. Fig. 22 shows the in-plane resistivity (resistivity along FeAs planes) data of the samples which exhibits sharp superconducting transition at $44 \mathrm{~K}$. The agreement of $T_{c}$ defined by two different techniques ensures the good quality of the obtained single crystals. The $T$-dependence of the resistivity exhibits $T^{\alpha}$ $(1<\alpha<1.2)$-dependence, reproducing the behavior observed in the polycrystalline samples (Fig.12) The $T_{c}$ values observed here are lower than that for polycrystalline samples, presumably due to the difference in the oxygen content, and/or intersubstitution between $\mathrm{O} /$ As.

Fig. 23 shows the in-plane resistivity under magnetic field $(H=1 \mathrm{~T} \sim 7 \mathrm{~T})$ with its direction perpendicular to the $a b$-plane $(H \perp a b)$ (Fig.M-4(a)), along the $a b$-plane and parallel to the current (Fig.23(b)), along the $a b$-plane and perpendicular to the current (Fig.4(c)), respectively. Application of magnetic field results in the decrease in $T_{c}$. Its effect is most prominent for $H \perp a b$ (Fig.23(a)), in which we observe significant broadening of the transition, characteristic feature of anisotropic (layered) superconductors.

From the resistivity data, one can roughly estimate the anisotropic upper critical field $\left(H_{c 2}\right)$. Here we define $H_{c 2}(\mathrm{~T})$ at each temperature as a magnetic field where the resistivity drops down to certain percentage compared to the (linearly extrapolated) normal state values. In Fig.24, we show $T$-dependence of so-defined $H_{c 2}(\mathrm{~T})$ with several resistivity-drop percentages (resisitvity-drop \%). When $H / / a b$ (Fig. 24(b)), $H_{c 2}{ }^{a b}$ 
is linear to $T$ and independent of the resistivity-drop percentage. On the other hand, for $H \perp a b$ (or $H / / c$ ) (Fig 24(a)), $H_{c 2}{ }^{c}$ shows non-linear behavior, as well as marked dependence on the resistivity-drop percentage, reflecting the resistivity broadening.

The fundamental parameter to characterize the anisotropic superconductors is the ratio of the upper critical fields, $\Gamma=\left(H_{c 2}{ }^{a b}(0) / H_{c 2}{ }^{c}(0)\right)$, termed as anisotropic parameter. One can obtain $\Gamma$ by taking the ratio of the slopes $\left(\mathrm{d} H_{c 2} / \mathrm{d} T\right)$ shown in Fig.24c- (a) and (b), since $H_{c 2}{ }^{a b}(0)$ is proportional to $\mathrm{d} H_{c 2} / \mathrm{d} T$. Calculated $\Gamma$ using various resistivity-drop $\%$ is about 5 , independent of the definition. The value is much smaller than cuprates [46], indicating that the superconductivity is more three dimensional. It is slightly larger than Ba-122 system $(\Gamma \sim 2)[47]$, presumably due to the different character of the counter layers $(\operatorname{LnO}$ vs. $\mathrm{Ba})$ which makes their anisotropy distinct from each other.

Jia et al. have carried out the resistivity measurements on $\mathrm{NdFeAsO}_{1-\mathrm{x}} \mathrm{F}_{\mathrm{x}}$ single crystals and estimated $\Gamma \sim 4.9-4.34$ [48]. Balicas et al. have done torque magnetometry measurements on $\mathrm{SmFeAsO}_{1-\mathrm{x}} \mathrm{F}_{\mathrm{x}}$ single crystals and reported $\Gamma \sim 9$ [49]. The estimated $\Gamma \sim 5$ in this study is comparable to those results. It should be noted that the samples used in their studies are much smaller (plate like with $\sim 30-100 \mu \mathrm{m}^{2}$ size) compared to the present study. The success of the growth of large single crystals opens new avenues in exploring more deeper physics on the Fe-based superconductors.

\section{The physical properties of $A M_{2} X_{2}$ samples}

\section{$4.1(\mathrm{Ca}, \mathrm{Na}) \mathrm{Fe}_{2} \mathrm{As}_{2}$}


HP synthesis technique is also effective in growing polycrystalline and single crystals of ' 122 ' phase. Fig. 25 shows the powder XRD patterns of the samples with nominal compositions of $\left(\mathrm{Ca}_{1-\mathrm{x}} \mathrm{Na}_{\mathrm{x}}\right) \mathrm{Fe}_{2} \mathrm{As}_{2}(x=0,0.2$ and 0.4$)$. The $\mathrm{ThCr}_{2} \mathrm{Si}_{2}$-type crystal structure $(\mathrm{I} 4 / \mathrm{mmm})$ is formed as the main phase for all samples. For $x=0-0.4$, majority of the apparent peaks can be indexed based on the $\mathrm{BaFe}_{2} \mathrm{As}_{2}[50]$ type crystal structure. Peaks due to impurity phases like FeAs, $\mathrm{CaO}$ and unknown phase(s) appeared as shown in Fig. 25.

The temperature dependence of the magnetic susceptibility $(\chi)$ of $\left(\mathrm{Ca}_{1-\mathrm{x}} \mathrm{Na}_{\mathrm{x}}\right) \mathrm{Fe}_{2} \mathrm{As}_{2}$ is shown in Fig.26. The clear drop in $\chi$, corresponding to the onset of superconductivity, is observed for $x=0.2$ and 0.4 samples. Superconducting transition temperatures determined onset of susceptibility, $T_{c}-\chi$ (onset), are 14.0 and $17.2 \mathrm{~K}$ for $x=0.2$ and 0.4 , respectively. The volume fractions estimated from the magnitude of shielding signals at $5 \mathrm{~K}$ is about 4 and $48 \%$ for $x=0.2$ and 0.4 , respectively. The volume fraction for $x=0.4$ is large enough as bulk superconductivity. We note that the samples for $x=0$ and 0.2 has large magnetic moment above $T_{\mathrm{c}}$, due to ferromagnetic impurities, most likely Fe.

We note that whole synthesis and characterization have been carried out within three days, thanks to the high throughput of HP synthesis technique for searching new 
superconductors. We also note that the presented data are on the first samples we have obtained. Further optimization should improve the sample quality.

\section{$4.2(\mathrm{Ba}, \mathrm{K}) \mathrm{Fe}_{2} \mathrm{As}_{2}$}

The parent and $\mathrm{K}$-doped $\mathrm{BaFe}_{2} \mathrm{As}_{2}$ crystallize with the $\mathrm{ThCr}_{2} \mathrm{Si}_{2}[51,52]$ structure, in tetragonal space group symmetry $I 4 / \mathrm{mmm}($ No. $139 ; \mathrm{Z}=2$ ) at room temperature and X-ray diffraction pattern is represented in Fig. 27. All observed reflections were indexed with tetragonal space group symmetry $14 / \mathrm{mmm}$. The lattice parameters of $\mathrm{BaFe}_{2} \mathrm{As}_{2}$ are $a=3.961(8) \AA$ and $c=12.996(1) \AA$, consistent with a report on a polycrystalline sample [51]. The refined lattice constants of $\mathrm{Ba}_{0.6} \mathrm{~K}_{0.4} \mathrm{Fe}_{2} \mathrm{As}_{2}$ are $a=3.914(2) \AA$ and $c=$ 13.305(1) A. Potassium doping results in a detectable shift in lattice parameters. The decrease $(1.20 \%)$ in the length of the $a$-axis, while the value of $c$-axis is increase $(2.37$ \%) which is consistent a report on polycrystalline prepared by using the solid state reaction method [53].

Fig. 28 shows $\chi$ in zero-field-cooled $(z f c)$ form for $\mathrm{Ba}_{0.6} \mathrm{~K}_{0.4} \mathrm{Fe}_{2} \mathrm{As}_{2}$ in 5 Oe applied field. Sharp drop in $\chi$ at $\sim 38 \mathrm{~K}$ is observed. The volume fractions estimated from the magnitude of shielding signals at $5 \mathrm{~K}$ is $\sim 70 \%$ for $x=0.4$, large enough as bulk superconductivity. So present result indicates that high pressure synthesis 
technique can be use for the synthesis of $\mathrm{BaFe}_{2} \mathrm{As}_{2}$ system and $\mathrm{K}$ - doping can be controlled as high pressure synthesis technique avoids the evaporation of K.

\section{3. $\mathrm{BaNi}_{2} \mathrm{P}_{2}$ single crystal}

In the case of $\mathrm{BaNi}_{2} \mathrm{P}_{2}$, a nickel-based pnictide superconductor with $T_{c}=2.5 \mathrm{~K}$, one can obtain single crystal samples by HP technique, which allows us to evaluate the anisotropic physical properties. Fig. 29 shows the temperature dependence of the in-plane resistivity $\left(\rho_{/ /}\right) . \rho_{/ /}$is about $80-100 \mu \Omega \mathrm{cm}$ at $300 \mathrm{~K}$ and shows T-linear dependence above 50K. Below 40K, T-dependence crosses over from T-linear to $T^{n}$ $(n \sim 3)$, then shows superconductivity at $T_{c} \sim 2.5 \mathrm{~K}$ (shown in inset). This value is consistent with previous report on polycrystalline samples[16]. Sharp superconducting transition and small residual resistivity $(<10 \mu \Omega \mathrm{cm})$ indicate the high quality of the single crystals obtained by HP synthesis method.

The resistivity across the $\mathrm{NiP}$ planes $\left(\rho_{\perp}\right)$ is about $500 \mu \Omega \mathrm{cm}$ at room temperature, and its $T$ - dependence is similar to that of $\rho_{/ /}$as shown in Fig. 29(b). The anisotropy $\rho_{\perp} / \rho_{/ /}$is about 5 , which is smaller than $A E \mathrm{Fe}_{2} \mathrm{As}_{2}(A E=\mathrm{Ba}, \mathrm{Sr}, \mathrm{Ca})[54-56]$, indicating three-dimensional charge transport of this system. 
Fig. 30(left) shows the $T$-dependence of $\rho_{/ /}$under magnetic field, $\mathrm{H} / /$ plane (upper one) and $\mathrm{H}_{\perp}$ plane (lower one) are shown respectively. Using the results, upper critical field is estimated and plotted in Fig. 30(right). In both $\mathrm{H} / /$ plane and $\mathrm{H}_{\perp}$ plane cases, upper critical field shows $T$-linear dependence near $T_{c}$. From the relational expression $\mathrm{Hc}_{2}(0)=0.69\left|\mathrm{dHc}_{2}(\mathrm{~T}) / \mathrm{dT}\right|_{\mathrm{T}=\mathrm{Tc}} T_{c}[57], \quad \mathrm{Hc}_{2}{ }^{\prime \prime}(0) /\left(\mathrm{Hc}_{2}{ }^{\perp}\right)(0) \quad$ can $\quad$ be estimated as $\sim 1600(650) \mathrm{Oe}\left(\xi_{/ /}\left(\xi_{\perp}\right) \sim 710(290) \AA\right)$. In the case of anisotropic metals, there is a relationship $\gamma=\mathrm{Hc}_{2}{ }^{\prime \prime}(0) / \mathrm{Hc}_{2}{ }^{\perp}(0)=\xi_{/ / /} \xi_{\perp}=\left(\mathrm{m}^{*}{ }_{\perp} / \mathrm{m}^{*} / /\right)^{1 / 2}$ where $\gamma$ is anisotropy factor. $\mathrm{Hc}_{2}{ }^{\prime \prime}(0) / \mathrm{Hc}_{2}{ }^{\perp}(0)$ of $\mathrm{BaNi}_{2} \mathrm{P}_{2}$ is $\sim 2.46$ and is comparable to the value of $\left(\rho_{\perp} / \rho_{/ /}\right)^{1 / 2} \sim 2.2$ obtained from the resistivity anisotropy, which means the physical property of $\mathrm{BaNi}_{2} \mathrm{P}_{2}$ is understood as anisotropic metal.

\section{Summary}

We introduce the HP synthesis of the iron (nickel)-based superconductors and their basic properties. We demonstrate the uniqueness of HP synthesis method in synthesizing iron (nickel)-based superconductors, including new materials and single crystals. Our systematic studies on the $\operatorname{LnFeAsO}$ system have revealed that $T_{c}$ is closely correlated with O-deficiency, synthesis pressure, variation of $L n$, physical pressure, shape of tetrahedron, etc, which points towards the importance of crystal structure for high- $T_{c}$ of 
the system. Measurement on single crystalline sample of $\mathrm{PrFeAsO}_{1-\mathrm{y}}$ reveals the superconducting anisotropy of $\sim 5$, which is smaller than cuprate based superconductors. As for 122-related systems, we can control the volatile elements and synthesis good quality samples. Also we have shown that HP technique is useful for growing single crystals of $\mathrm{BaNi}_{2} \mathrm{P}_{2}$.

History of cuprate superconductivity highlights the versatility of HP technique as a tool in exploring new materials. Using this technique, a number of new superconductors, including multi-layered cuprates, have been discovered. Multi-layer iron (nickel) based superconductors are still undiscovered. Stabilization and crystallization of multilayer systems can be achieved only by HP technique, with higher- $T_{c}$ might exist.

\section{Acknowledgments}

The authors are grateful to S. Ishida, M. Nakajima, S. Uchida, Y. Tanaka for transport properties measurements and helpful discussion. P.M.S. is grateful to the Japan Society for the Promotion of Science (JSPS) for the JSPS postdoctoral fellowship (2006-2008). This work was supported by Grant-in-Aid for Specially promoted Research (20001004) from The Ministry of Education, Culture, Sports, Science and Technology (MEXT) and JST, Transformative Research-Project on Iron Pnictides (TRIP). 


\section{References:}

[1] Y. Kamihara, T. Watanabe, M. Hirano, H. Hosono, J. Am. Chem. Soc. 130 (2008) 3296.

[2] H. Kito, H. Eisaki, A. Iyo, J. Phys. Soc. Jpn. 77(2008) 063707.

[3] G. F. Chen, Z. Li, G. Li, J. Zhou, D. Wu, J. Dong, W. Z. Hu, P. Zheng, Z. J. Chen, J.

L. Luo, N. L. Wang., Phys. Rev. Lett. 101(2008)057007; arXiv: 0803.0128.

[4] A. S. Sefat, M. A. McGuire, B. C. Sales, R. Jin, J.Y. Howe, D. Mandrus, Phys. Rev. B 77 (2008) 174503; arXiv:0803.2528.

[5] X. H. Chen, T. Wu, G. Wu, R. H. Liu, H. Chen, D. F. Fang, Nature 453 (2008) 761; arXiv: 0803.3603.

[6] G. F. Chen, Z. Li, D. Wu, G. Li, W.Z. Hu, J. Dong, P. Zheng, J.L. Luo, N.L. Wang. Phys. Rev. Lett. 100 (2008) 247002;arXiv: 0803.3790; G. F. Chen, Z. Li, D. Wu, J. Dong, G. Li, W. Z. Hu, P. Zheng, J. L. Luo, N. L. Wang, Chin. Phys. Lett. 25 (2008) 2235; arXiv: 0804.4384.

[7] Z.-A. Ren, J. Yang, W. Lu, W. Yi, X.-L. Shen, Z.-C. Li, G.-C. Che, X.-L. Dong, L.-L. Sun, F. Zhou, Z.-X. Zhao, Europhys. Lett. 82(2008)57002; arXiv: 0803.4234.

[8] K. Miyazawa et al. J. Phys. Soc. Jpn. 78 (3)(2009) In press. 
[9] Z.-A. Ren, G.-C. Che, X.-L. Dong, J. Yang, W. Lu, W. Yi, X.-L. Shen, Z.-C. Li, L.-L. Sun, F. Zhou, Z.-X. Zhao, Europhys. Lett. 83 (2008)17002; arXiv: 0804.2582.

[10] L. Wei, X.-L. Shen, J. Yang, Z.-C. Li, W. Yi, Z.-A. Ren, X.-L. Dong, G.-C. Che, L.-L. Sun, F. Zhou, Z.-X. Zhao, Sol. State Comm. 148 (2008) 168; arXiv: 0804.3725.

[11] J. Yang, Z.-C. Li, W. Lu, W. Yi, X.-L. Shen, Z.-A. Ren, G.-C. Che, X.-L. Dong, L.-L. Sun, F. Zhou, Z.-X. Zhao, Supercond. Sci. Technol. 21(2008) 082001; arXiv: 0804.3727 .

[12] P. M. Shirage, K. Miyazawa, H. Kito, H. Eisaki, A. Iyo, Phys. Rev. B. 78 (2008) 172503.

[13] P. M. Shirage, K. Miyazawa, H. Kito, H. Eisaki, A. Iyo, App. Phys. Exp. 1 (2008) 081702.

[14] T. Watanabe, H. Yanagi, Y. Kamihara, T. Kamiya, M. Hirano, H. Hosono, J. Solid State Chem. 181 (2008)2117.

[15] F.-C. Hsu, J.-Y. Luo, K.-W. Yeh, T.-K. Chen, T.-W. Huang, P. M. Wu, Y.-C. Lee, Y.-L. Huang, Y.-Y. Chu, D.-C. Yan, M.-K. Wu, Proc. Natl. Acad. Sci. U.S.A. 105(2008) 14262.

[16] T. Mine, H. Yanagi, T. Kamiya, Y. Kamihara, M. Hirano, H. Hosono, Solid State Comm., 147(2008) 111. 
[17] P. M. Shirage, D. D. Shivagan, Y. Tanaka, Y. Kodama, H. Kito, A. Iyo, Appl. Phys. Lett. 92(2008)222501.

[18] H. Yamauchi, M. Karppinen, Supercond. Sci. Technol. 13 (2000) R33.

[19] E. Takayama-Muromachi, T. Kawashima, M. Isobe, Y. Matsui, Physica C 235-240 (1994)987.

[20] Z. Hiroi, M. Takano, Physica C 235-240 (1994 )29.

[21] H. Ihara, K. Tokiwa, H. Ozawa, M. Hirabayashi, H. Matuhata, A. Negishi, Y. S. Song, Jpn. J. Appl. Phys. 33 (1994)L300.

[22] Z.-A. Ren, J. Yang, W. Lu, W. Yi, G.-C. Che, X.-L. Dong, L.-L. Sun, Z.-X. Zhao, Materials Research Innovations 12 (2008) 105 ; arXiv:0803.4283.

[23] Z.-A. Ren, W. Lu, J. Yang, W. Yi, X.-L. Shen, Z.-C. Li, G.-C. Che, X.-L. Dong, L.-L. Sun, F. Zhou, Z.-X. Zhao, Chin. Phys. Lett. 25 (2008) 2215; arXiv:0804.2053.

[24] K. Miyazawa, H. Eisaki, H. Kito, P. M. Shirage, K. Kihou, M. Ishikado, A. Iyo, J. Phys. Soc. Jpn.(Supplement C) 77 (2008) 127.

[25]N. Ni, S. Nandi, A. Kreyssig, A. I. Goldman, E. D. Mun, S. L. Bud'ko, P. C. Canfield, Phys. Rev. B 78 (2008) 014523.

[26]N. Ni, S. L. Bud'ko, A. Kreyssig, S. Nandi, G. E. Rustan, A. I. Goldman, S. Gupta, J. D. Corbett, A. Kracher, P. C. Canfield, Phys. Rev. B 78 (2008) 014507. 
[27] J.-Q. Yan, A. Kreyssig, S. Nandi, N. Ni, S. L. Bud'ko, A. Kracher, R. J. McQueeney, R. W. McCallum, T. A. Lograsso, A. I. Goldman, P. C. Canfield, Phys. Rev. B 78 (2008)024516.

[28]C.H. Lee, A. Iyo, H. Eisaki, H. Kito, M.T. Fernandez-Diaz, T. Ito, K. Kihou, H. Matsuhata, M. Braden, and K. Yamada, J. Phys. Soc. Jpn. 77 (2008) 083704.

[29] P. Cheng, L. Fang, H. Yang, X. Zhu, G. Mu, H. Luo, Z. Wang, H.-H. Wen, Science in China G 51(6) (2008) 719.

[30] N. Takeshita, A. Iyo, H. Eisaki, H. Kito, T. Ito, J. Phys. Soc. Jpn. 77 (2008) 075003.

[31] N. Takeshita, T. Yamazaki, A. Iyo, H. Eisaki, H. Kito, T. Ito, K. Hirayama, H. Fukazawa, Y. Kohori, J. Phys. Soc. Jpn. 77 (2008) Supplement C pp. 131.

[32] N. Môri, H. Takahashi, N. Takeshita: High Pressure Res. 24 (2004) 225.

[33] K. Murata, K. Yokogawa, H. Yoshino, S. Klotz, P. Munsch, A. Irizawa, M. Nishiyama, K. Iizuka, T. Nanba, T. Okada, Y. Shiraga, S. Aoyama, Rev. Sci. Instrum. 79 (2008) 085101.

[34] H. Takahashi, K. Igawa, K. Arii, Y. Kamihara, M. Hirano, and H. Hosono, Nature 453 (2008) 376. 
[35]H. Okada, K. Igawa, H. Takahashi, Y. Kamihara, M. Hirano, H. Hosono, K. Matsubayashi, Y. Uwatoko, J. Phys. Soc. Jpn. 77 (2008) 113712.

[36] K. Igawa, H. Okada, H. Takahashi, S. Matsuishi, Y. Kamihara, M. Hirano, H. Hosono, K. Matsubayashi, Y. Uwatoko, arXiv:0810.1377.

[37] M. S. Torikachvili, S. L. Bud'ko, N. Ni, and P. C. Canfield, Phys. Rev. Lett. 101 (2008) 057006.

[38] T. Park, E. Park, H. Lee, T. Klimczuk, E. D. Bauer, F. Ronning, J. D. Thompson, J. Phys.: Condens. Matter 20 (2008) 322204.

[39] H. Fukazawa, N. Takeshita, T. Yamazaki, K. Kondo, K. Hirayama, Y. Kohori, K. Miyazawa, H. Kito, H. Eisaki, and A. Iyo, J. Phys. Soc. Jpn. 77 (2008) 105004.

[40] H. Kotegawa, H. Sugawara, H. Tou, J. Phys. Soc. Jpn. 78 (2009) 013709.

[41] W. Yu, A. A. Aczel, T. J. Williams, S. L. Bud'ko, N. Ni, P. C. Canfield, G. M. Luke, arXiv:0811.2554, to be published in Phys. Rev. B.

[42]K. Matsubayashi, private communication.

[43] D. J. Singh, M.-H. Du, Phys. Rev. Lett. 100 (2008) 237003; V. Vildosola, L. Pourovskii, R. Arita, S. Biermann, A. Georges, Phys. Rev. B 78 (2008) 064518.

[44] R. Kumai, N. Takeshita, C.-H. Lee, T. Ito, H. Kito, A. Iyo and H. Eisaki, J. Phys. Soc. Jpn. 78 (2009) 013705. 
[45] M. Ishikado et al. Accepted (Physica C ISS2008 proceeding).

[46] S. Tajima, G. D. Gu, S. Miyamoto, A. Odagawa, N. Koshizuka, Phys. Rev. B 48 (1993) 16164.

[47] H. Q. Yuan, J. Singleton, F. F. Balakirev, G. F. Chen, J. L. Luo, N. L. Wang, arXiv:0807.3137(2008).

[48] Y. Jia, P. Cheng, L. Fang, H. Luo, H. Yang, C. Ren, L. Shan, C. Gu, H.-H. Wen, App. Phys. Lett. 93 (2008) 032503.

[49] L. Balicas, A. Gurevich, Y. J. Jo, J. Jaroszynski, D. C. Larbalestier, R. H. Liu, H. Chen, X. H. Chen, N. D. Zhigadlo, S. Katrych, Z. Bukowski, J. Karpinski, arXiv:0809.4223v1(2008); J. Jaroszynski, F. Hunte, L. Balicas, Y. -J. Jo, I. Raijcevi'c, A. Gurevich, D. C. Larbalestier, arXiv:0810.2469v1(2008).

[50] M. Pfisterer, G. Nagorsen, Z. Naturforsch. B:Chem. Sci. 35 (1980) 703.

[51] S. Rozsa, H. U. Schuster, Z. Naturforsch. B: Chem. Sci. 36 (1981) 1668.

[52] A. Czybulka, M. Noak, H.-U. Schuster, Z. Anorg. Allg. Chem. 609 (1992) 122.

[53] M. Rotter, M. Pangerl, M. Tegel, D. Johrendt, Angew. Chem. Int. Ed. 47 (2008) 7949.

[54] X. F. Wang, T. Wu, G. Wu, H. Chen, Y. L. Xie, J. J. Ying, Y. J. Yan, R. H. Liu, X.H. Chen, cond-mat, arXiv0806.2452.

[55] G. F. Chen, Z. Li, J. Dong, G. Li, W.Z. Hu, X.D. Zhang, X.H. Song, P. Zheng, N. L. Wang, J. L. Luo, Phys. Rev. B 78 (2008)224512; cond-mat, arXiv0806.2648. 
[56] G. Wu, H. Chen, T. Wu, Y. L. Xie, Y. J. Yan, R. H. Liu, X. F. Wang, J. J. Ying, X.

H. Chen, J. Phys.:Cond. Matt. 20 (2008) 422201; cond-mat, arXiv0806.4279.

[57] N.R. Werthamer, E. Helfand and P.C. Hohenberg, Phys. Rev. 147 (1966) 295.

\section{Figure captions:}

Fig. 1 (a) Photo graph of wedge-type cubic-anvil, high pressure apparatus (b) a schematic illustration of the sample cell assembly used in high-pressure synthesis technique.

Fig. 2 Flowchart of process involved in HP technique. Here we represent the example of $\operatorname{LnFeAsO} \mathrm{F}_{1-y}$ system.

Fig 3. Powder $\mathrm{XRD}$ patterns of O-deficient $\mathrm{NdFeAsO}_{1-\mathrm{y}}$ (with nominal compositions $1-y=0.70-0.85)$ samples synthesized by HP technique.

Fig. 4 The powder XRD patterns of oxygen-deficient $\mathrm{NdFeAsO}_{1-\mathrm{y}}$ (with nominal compositions $1-y=0.70-0.85$ ) samples synthesized by high-pressure technique.

Fig. 5 The temperature-dependence of magnetic susceptibility of oxygen-deficient $\mathrm{NdFeAsO}_{1-y}$ (with compositions $1-y=0.84-0.96$ ) samples synthesized by HP technique. The superconducting volume fraction of the sample $1-y=0.84$ estimated from the magnetic susceptibility at $\mathrm{T}=5 \mathrm{~K}$ (zero-field cooling) is about $90 \%$. The sample $1-y=0.84$ contains ferromagnetic minor phases more, so the background of susceptibility is 
different from the other samples.

Fig. 6 Temperature-dependence of the resistivity of O-deficient $\mathrm{NdFeAsO}_{1-\mathrm{y}}$ (with compositions $1-\mathrm{y}=0.80-0.96$ ) samples synthesized by HP technique.

Fig. 7 The electronic phase diagram of oxygen-deficient $\mathrm{NdFeAsO}_{1-\mathrm{y}}$ samples synthesized by high-pressure technique. The oxygen content estimated from the result of the neutron diffraction experiments is also shown. The $a$-axis length decreases as the oxygen deficiency increases, and superconductivity seems to develop discontinuously below a 3.964A.

Fig. 8 (a) Powder XRD patterns for the samples with a nominal composition of $\mathrm{DyFeAsO}_{0.7}$ synthesized at various pressures ranging from 2.0 to $5.5 \mathrm{GPa}$. (b) The enlargement of (212) peaks around 2 (deg.) $=57 \sim 58(\mathrm{~b})$.

Fig. 9 Temperature dependence of susceptibility for the samples with a nominal composition of $\mathrm{DyFeAsO}_{0.7}$ synthesized at pressures of $3.5,4.5$ and $5.5 \mathrm{GPa} . T_{\mathrm{c}}$ 's are shown by arrows.

Fig. 10 The plot $T_{c}$ of $L n \mathrm{FeAsO}_{1-\mathrm{y}}(\mathrm{Ln}=\mathrm{Sm}, \mathrm{Gd}, \mathrm{Tb}$ and $\mathrm{Dy}$ ) samples against the $a$-axis lattice parameter at various pressures.

Fig. 11 Temperature (T)- dependence of the magnetic susceptibility of the $\mathrm{LnFeAsO}_{1-y}$ samples for $L n=\mathrm{La}, \mathrm{Ce}, \mathrm{Pr}, \mathrm{Nd}, \mathrm{Sm}, \mathrm{Gd}, \mathrm{Tb}$ and $\mathrm{Dy} . T_{\mathrm{c}}($-onset) are indicated by 
arrows.

Fig. 12 Temperature $(T)$ - dependence of the resistivity of the $L n \mathrm{FeAsO}_{1-\mathrm{y}}$ samples for $L n=\mathrm{La}, \mathrm{Ce}, \mathrm{Pr}, \mathrm{Nd}, \mathrm{Sm}, \mathrm{Gd}, \mathrm{Tb}$ and $\mathrm{Dy}$. The insets show the $T$-dependence of resistivity near $T_{\mathrm{c}}$ and the definition of $T_{\mathrm{c}}$ ( -onset).

Fig. 13 The plot $T_{c}$ of $L n \mathrm{FeAsO}_{1-\mathrm{y}}$ samples against the $a$-axis lattice parameter, sample prepared by high pressure synthesis technique.

Fig. 14 The pressure dependence of the $T_{c}$ of $\mathrm{NdFeAsO}_{0.6}$ and $\mathrm{NdFeAsO}_{0.8}$ samples. Inset Fig. 14 shows the temperature dependence of the resistivity of $\mathrm{NdFeAsO}_{0.8}$ sample under different pressure.

Fig. 15 shows the temperature dependence of the resistivity of $\mathrm{NdFeAsO}_{0.85}$ sample under different pressure.

Fig. 16 shows the pressure dependence of the relative lattice constants of $\mathrm{NdFeAsO}_{0.7}$ (nominal composition). The open marks show that the experiments were performed in the pressure region where the pressure-transmitting medium is frozen and uniaxial effect may be included.

Fig. 17 shows the pressure dependence of the crystal structure parameters of $\mathrm{NdFeAsO}_{0.7}$ (nominal composition). The open marks show that the experiments were performed in the pressure region where the pressure-transmitting medium is frozen and 
uniaxial effect may be included.

Fig. 18 The relation between alpha and $T_{c}$ of $L n \mathrm{FeAsO}_{1-y}(L n=\mathrm{La}$ and $\mathrm{Nd})$.

Fig. 19 The relation between alpha and $T_{c}$ of Iron based superconductors. $T_{c}$ approaches to maximum when $\mathrm{FeAs}_{4}$-tetrahedrons forms a perfect regular in which -corresponds to $109.47^{\circ}$.

Fig. 20 Photograph of single crystal $\mathrm{PrFe} \mathrm{AsO}_{0.7}$ grown by high-pressure method. Length scale is presented in the arrow.

Fig. 21 Magnetic susceptibility as a function of temperature on $\mathrm{PrFeAsO}_{0.7}$ single crystals.

Fig. 22 Resistivity as a function of temperature data on $\mathrm{PrFeAsO}_{0.7}$ single crystal.

Fig. 23 Resistivity as a function of temperature under magnetic field (0-7T) for (a) $H \perp$ $a b$, (b) $H / / a b, \quad H \perp I$, and (c) $H / / a b / / I$.

Fig. 24 Temperature dependences of the upper critical field $\left(H_{c 2}\right)$ for (a) $H_{c 2} / / a b$ and (b) $H_{c 2} / / a b / / I$ for various cut percentages, $90 \%, 70 \%, 50 \%, 30 \%, 15 \%, 10 \%$, and $1 \%$, from onset( $100 \%$ ) to zero resistivity( $0 \%)$. (c) resistivity-drop dependence of $\Gamma$, derived from (a),(b).

Fig. 25 Powder x-ray diffraction patterns for the samples of $\mathrm{Ca}_{1-x} \mathrm{Na}_{x} \mathrm{Fe}_{2} \mathrm{As}_{2}$ with nominal compositions of $x=0,0.2$ and 0.4 .

Fig. 26 Temperature dependence of magnetic susceptibility for the samples of 
$\mathrm{Ca}_{1-x} \mathrm{Na}_{x} \mathrm{Fe}_{2} \mathrm{As}_{2}$ with nominal compositions of $x=0,0.2$ and 0.4 . Arrows in the figures show onsets of superconducting transitions.

Fig. $27 \mathrm{XRD}$ of $\mathrm{Ba}_{1-\mathrm{x}} \mathrm{K}_{\mathrm{x}} \mathrm{Fe}_{2} \mathrm{As}_{2}$ polycrystalline samples synthesized by high pressure technique.

Fig. 28 Temperature (T)- dependence of the magnetic susceptibility of the $\mathrm{Ba}_{1-x} \mathrm{~K}_{x} \mathrm{Fe}_{2} \mathrm{As}_{2}$ samples with $x=0.4$.

Fig. 29 (a)Temperature profile of the in-plane resistivity $\rho_{/ /}$for the $\mathrm{BaNi}_{2} \mathrm{P}_{2}$ single crystal. Inset shows the temperature below $45 \mathrm{~K}$. The dotted line is calculated results from $\rho=\rho_{0}+A T^{n}$ with $\rho_{0}=5.02 \mu \Omega \mathrm{cm}, A=5.29 \times 10^{-5} \mu \Omega \mathrm{cmK}^{-\mathrm{n}}$ and $n=3.02$. (b) Temperature profile of the out ofplane resistivity $\rho_{\perp}$ for the $\mathrm{BaNi}_{2} \mathrm{P}_{2}$ single crystal. Inset shows the temperature below $45 \mathrm{~K}$.

Fig. 30 (left) Temperature profiles of the in-plane resistivity for the parallel (upper) and perpendicular (lower) to plane with the transport current perpendicular to field. (right) The upper critical field of $\mathrm{BaNi}_{2} \mathrm{P}_{2}$ single crystal for both magnetic field parallel and perpendicular to plane. 
Figures

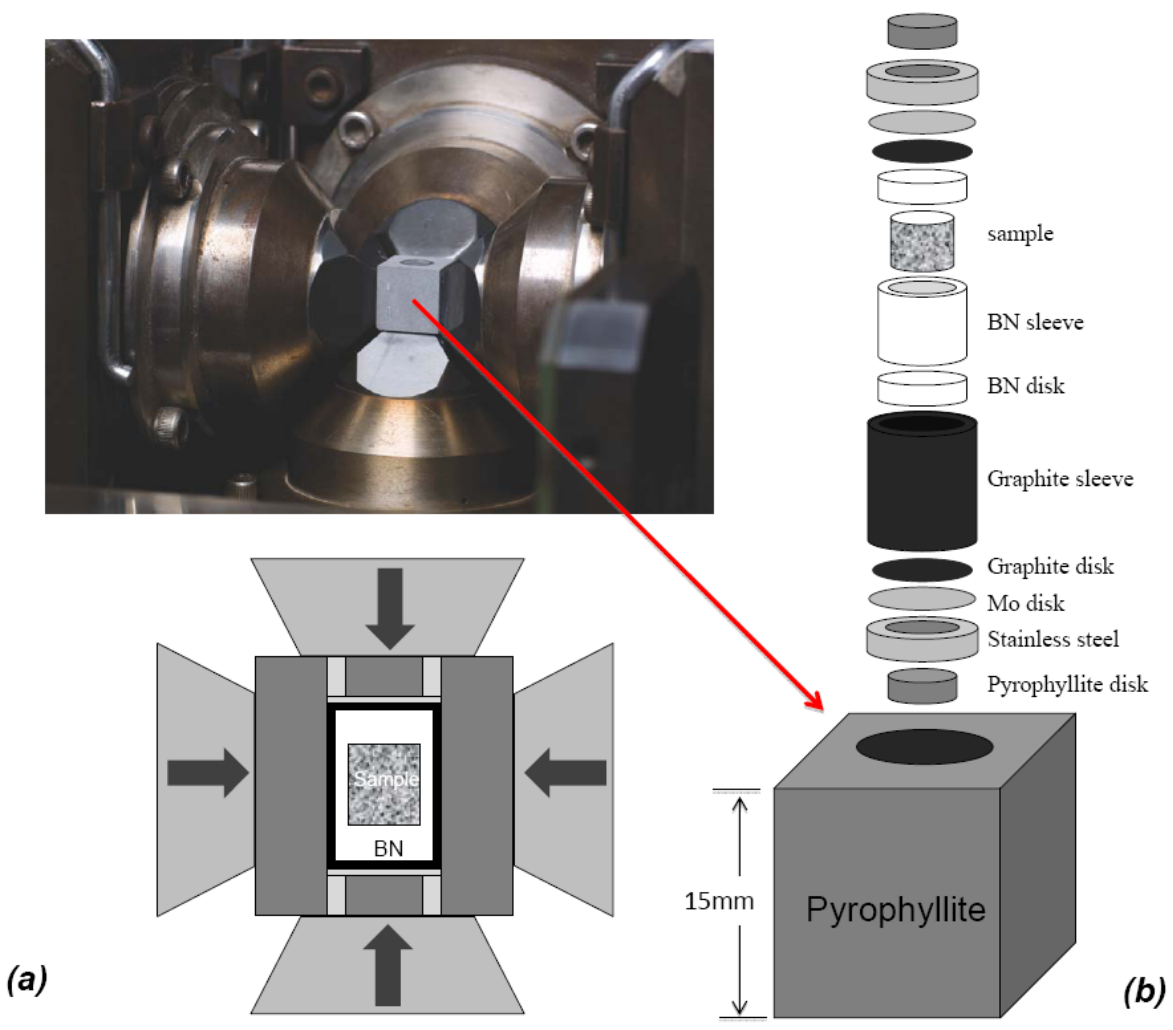

Shirage-Physica C-Figure 1 


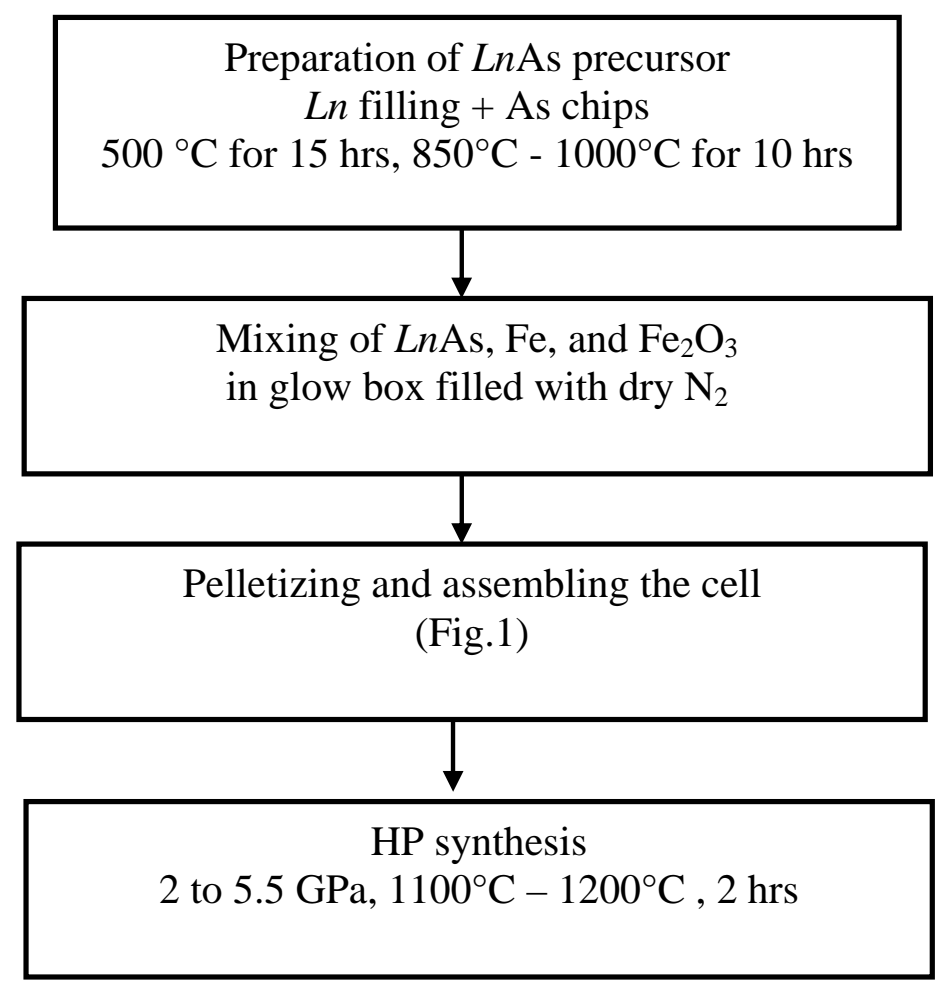

\section{Shirage-Physica C-Figure2}




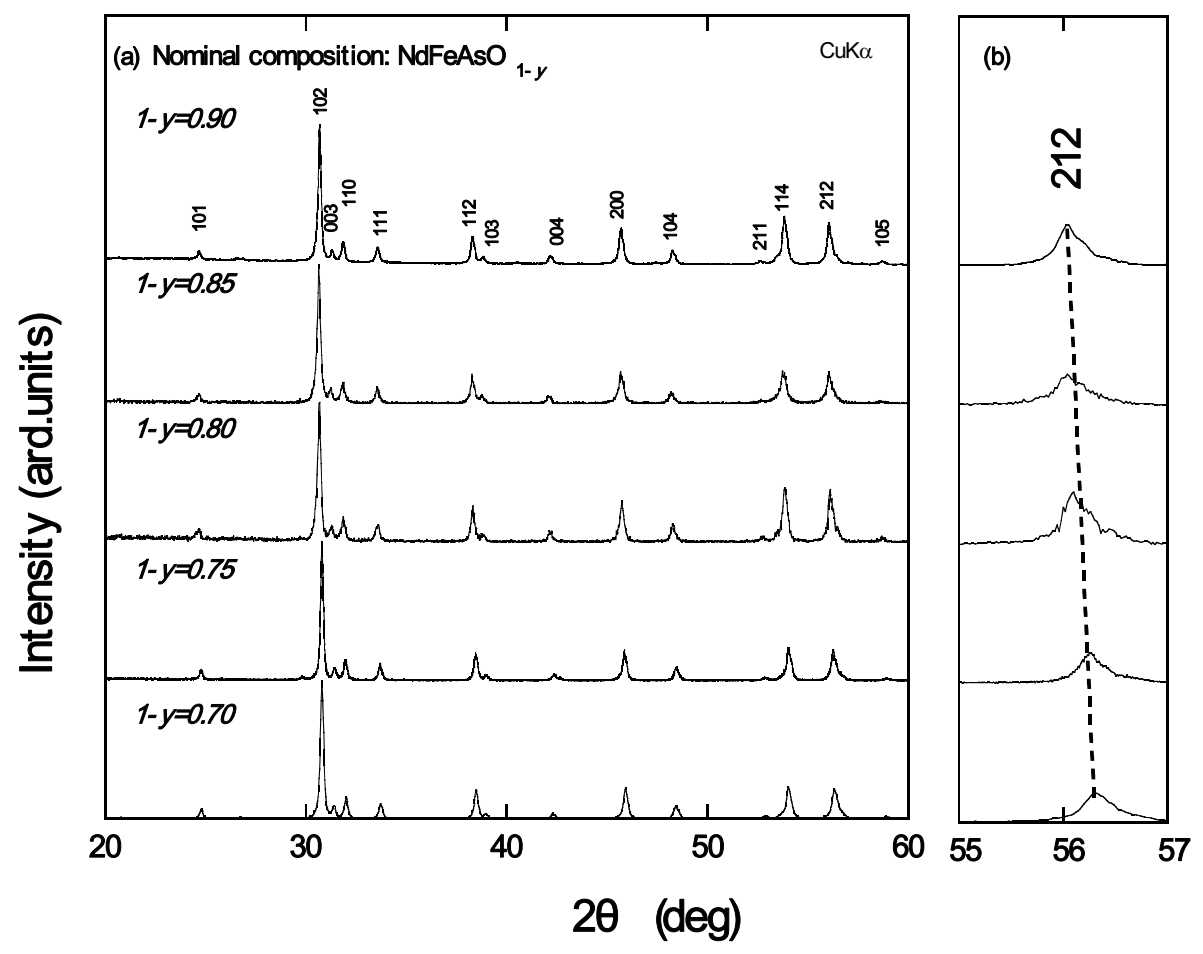

Shirage-Physica C-Figure3 


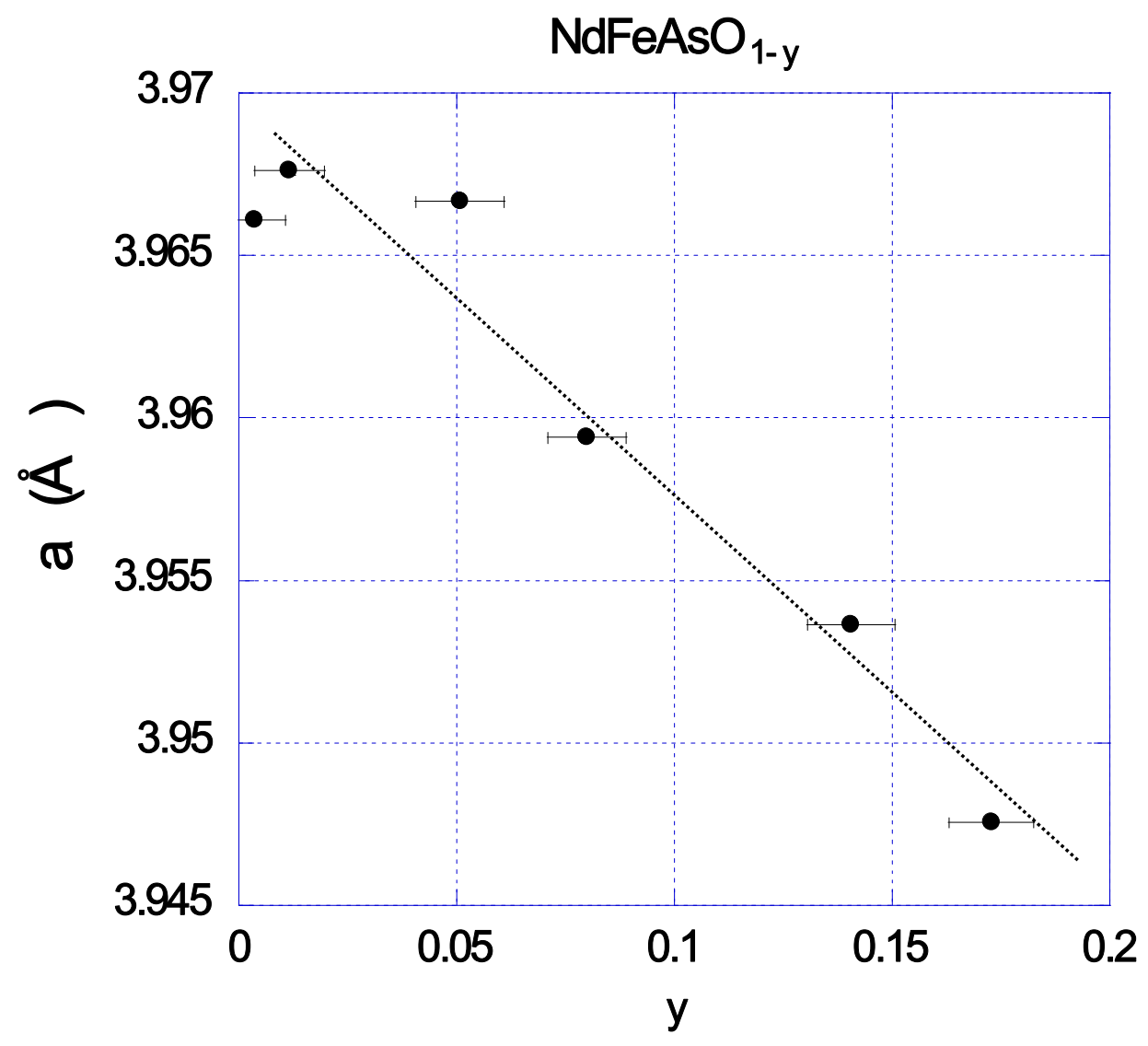

Shirage-Physica C-Figure4 


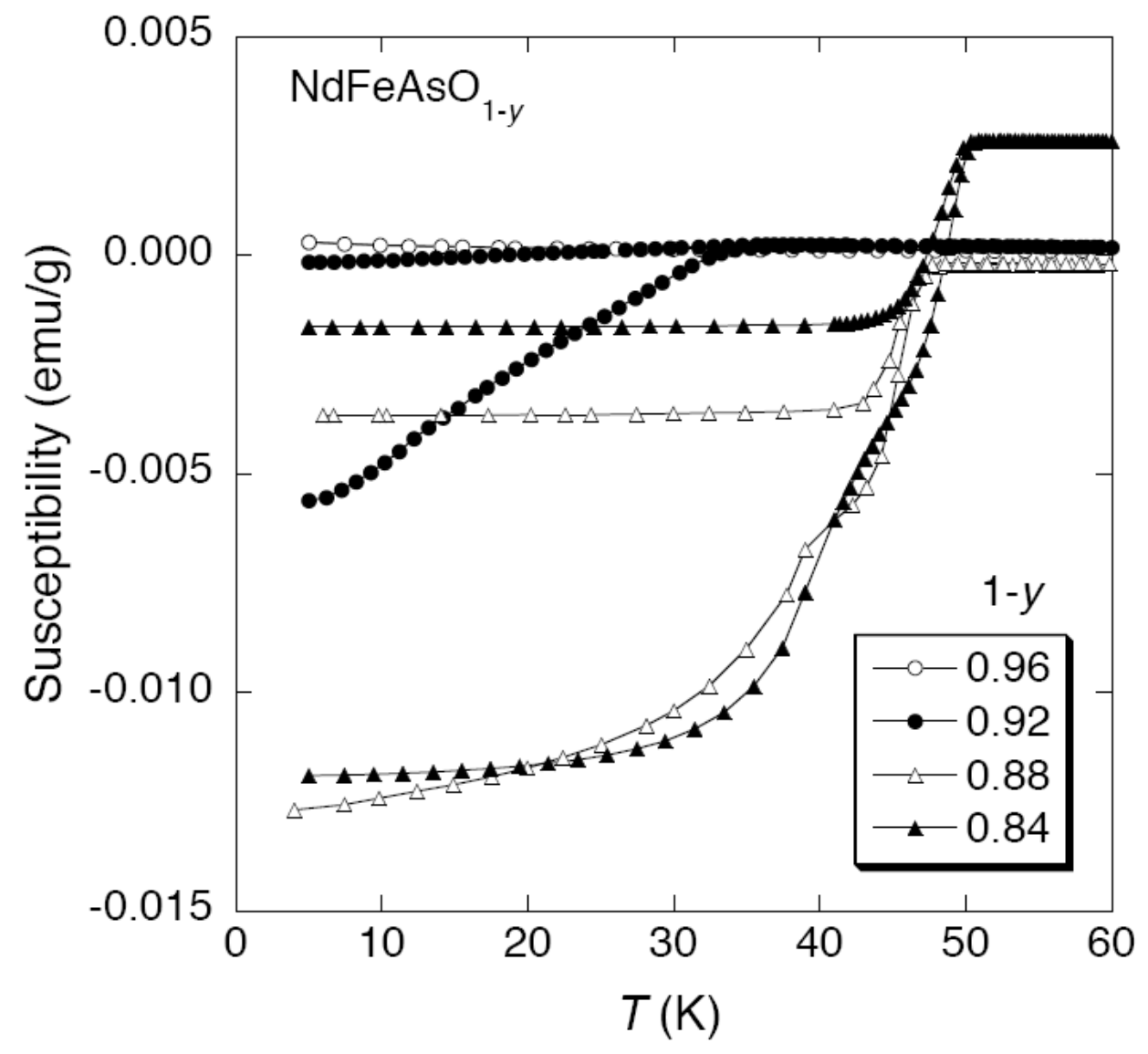

Shirage-Physica C-Figure5 


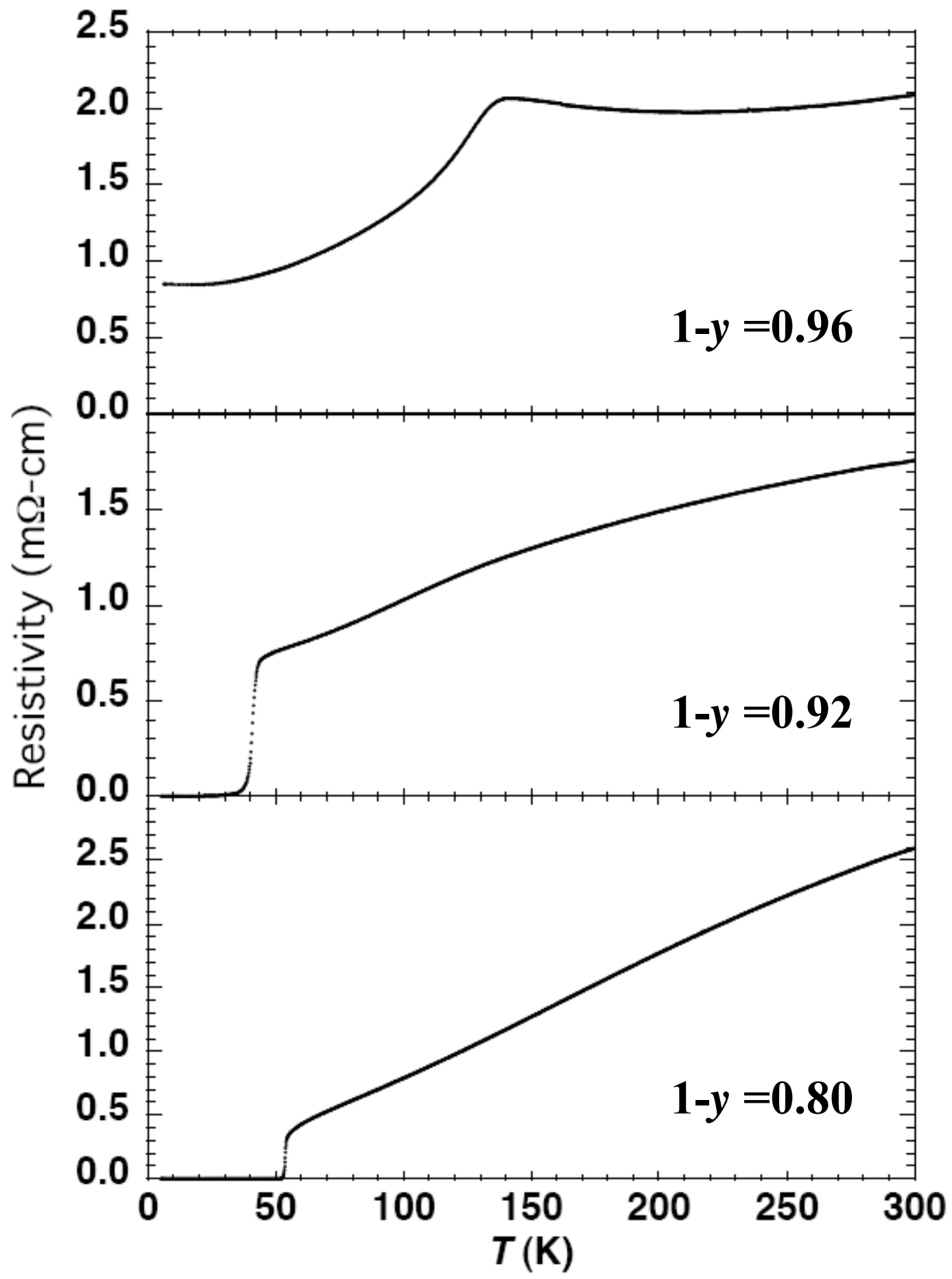

Shirage-Physica C-Figure 6 


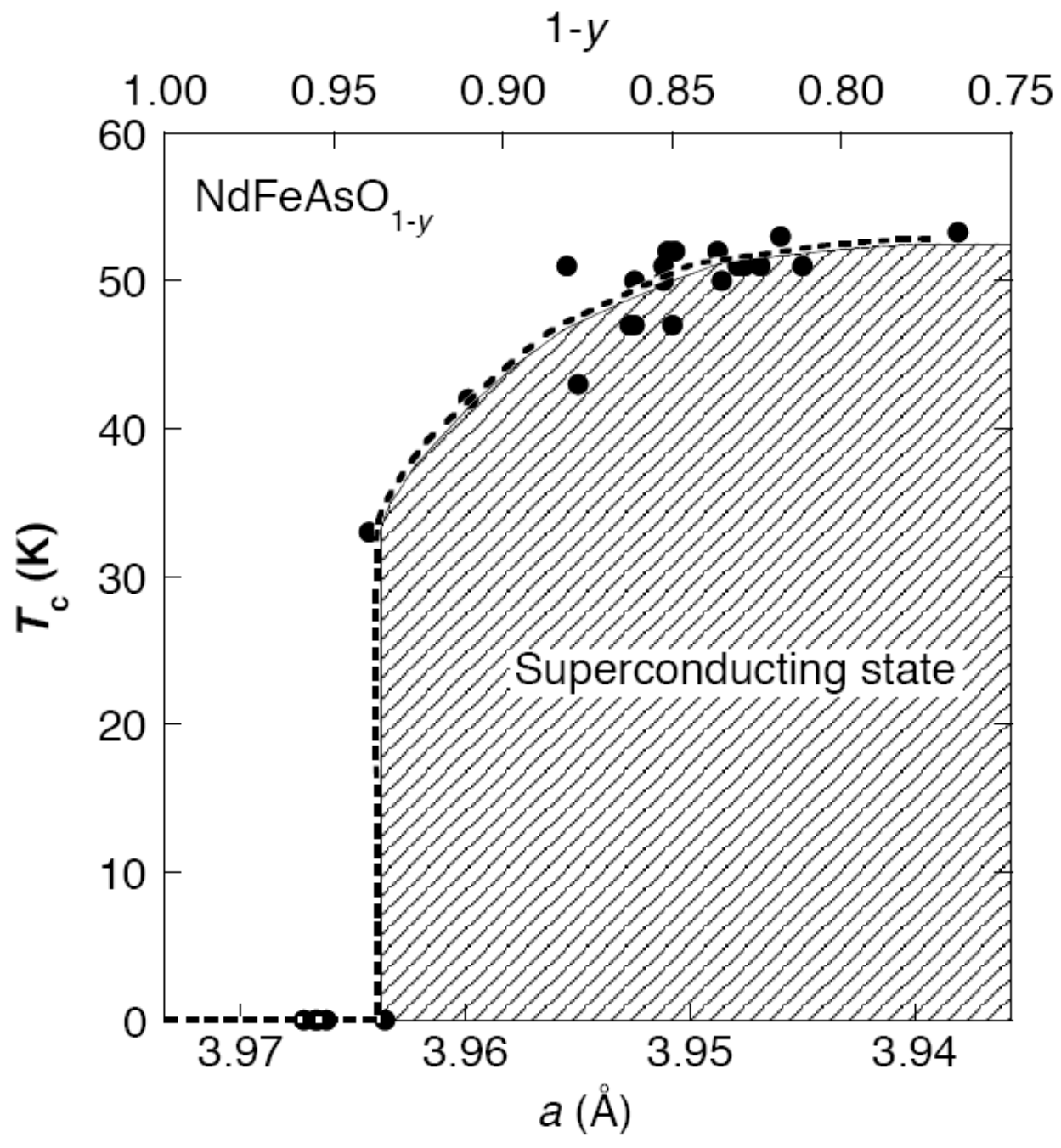

Shirage-Physica C-Figure7 


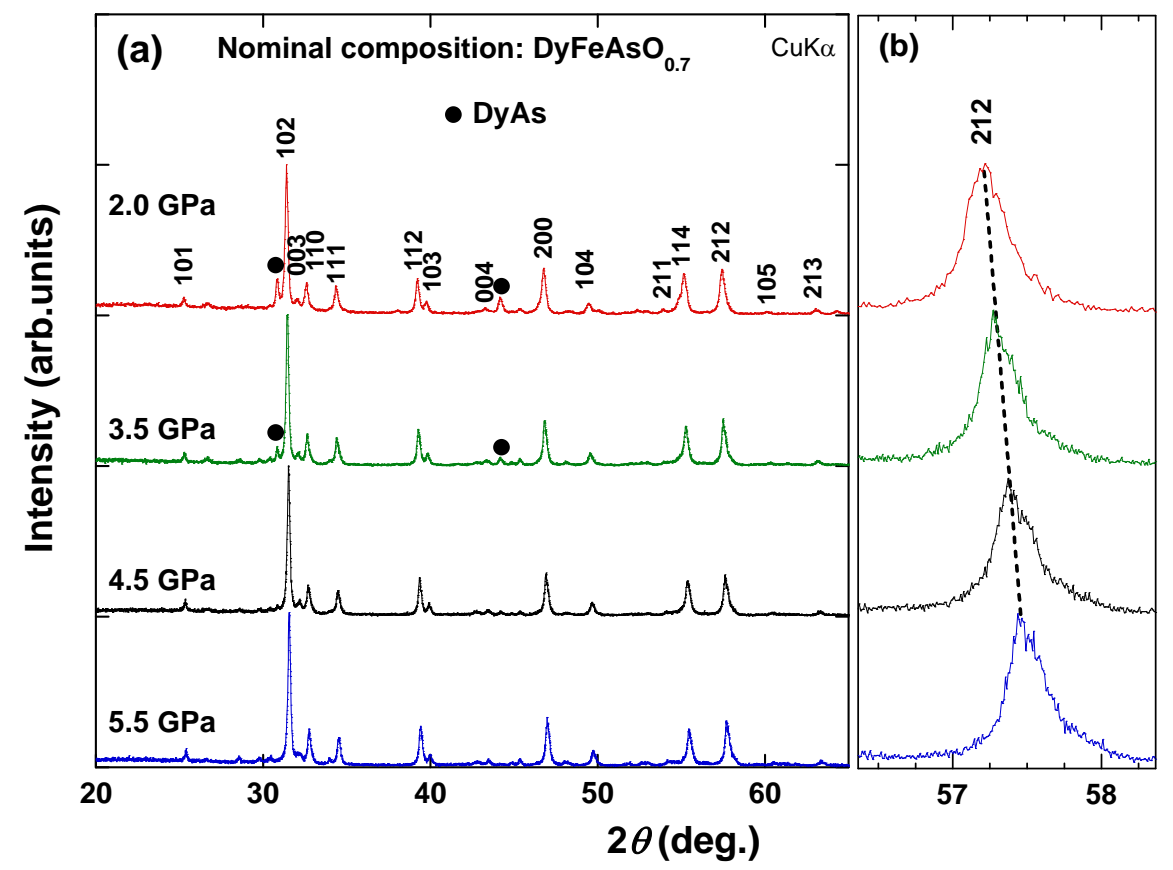

Shirage-Physica C-Figure8 


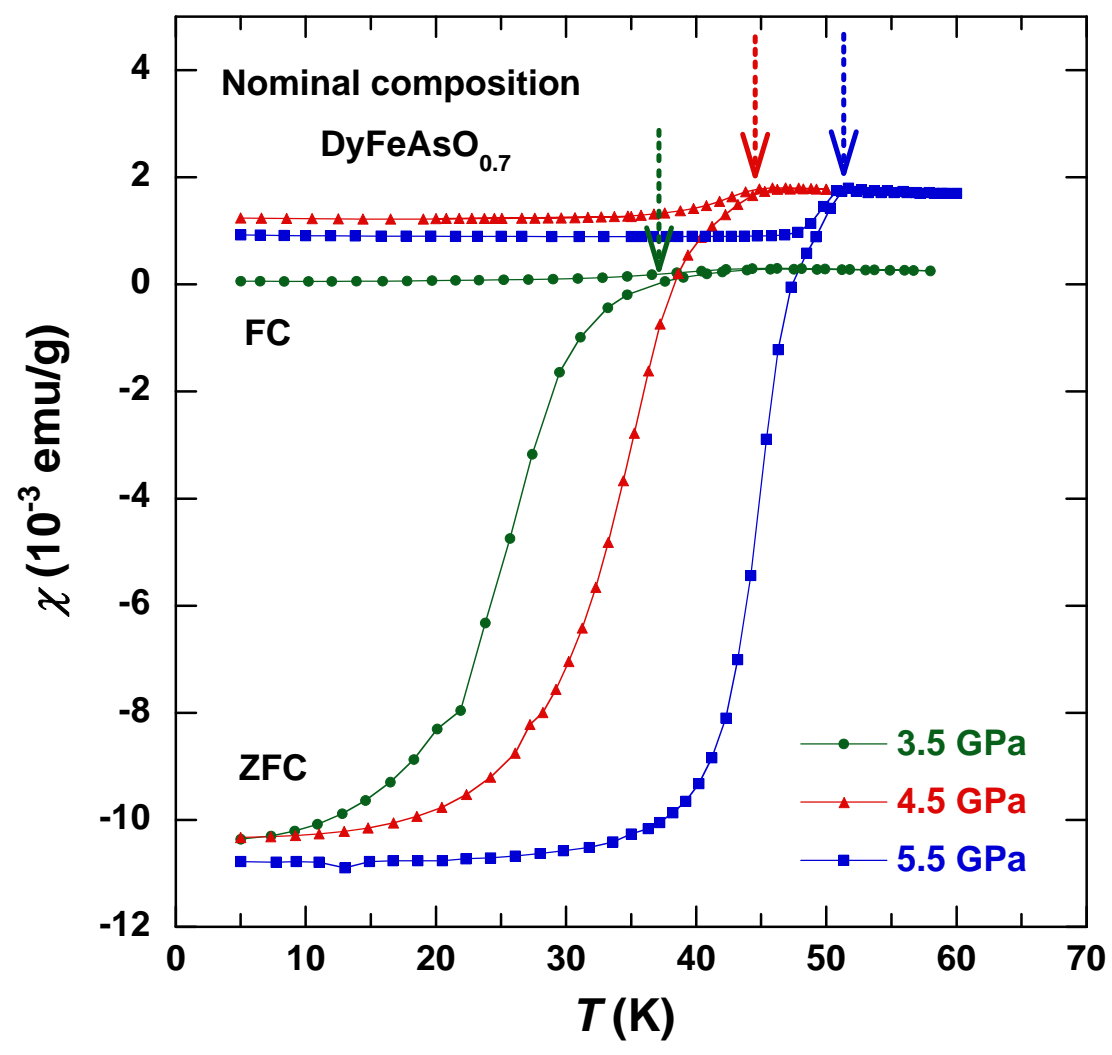

Shirage-Physica C-Figure9 


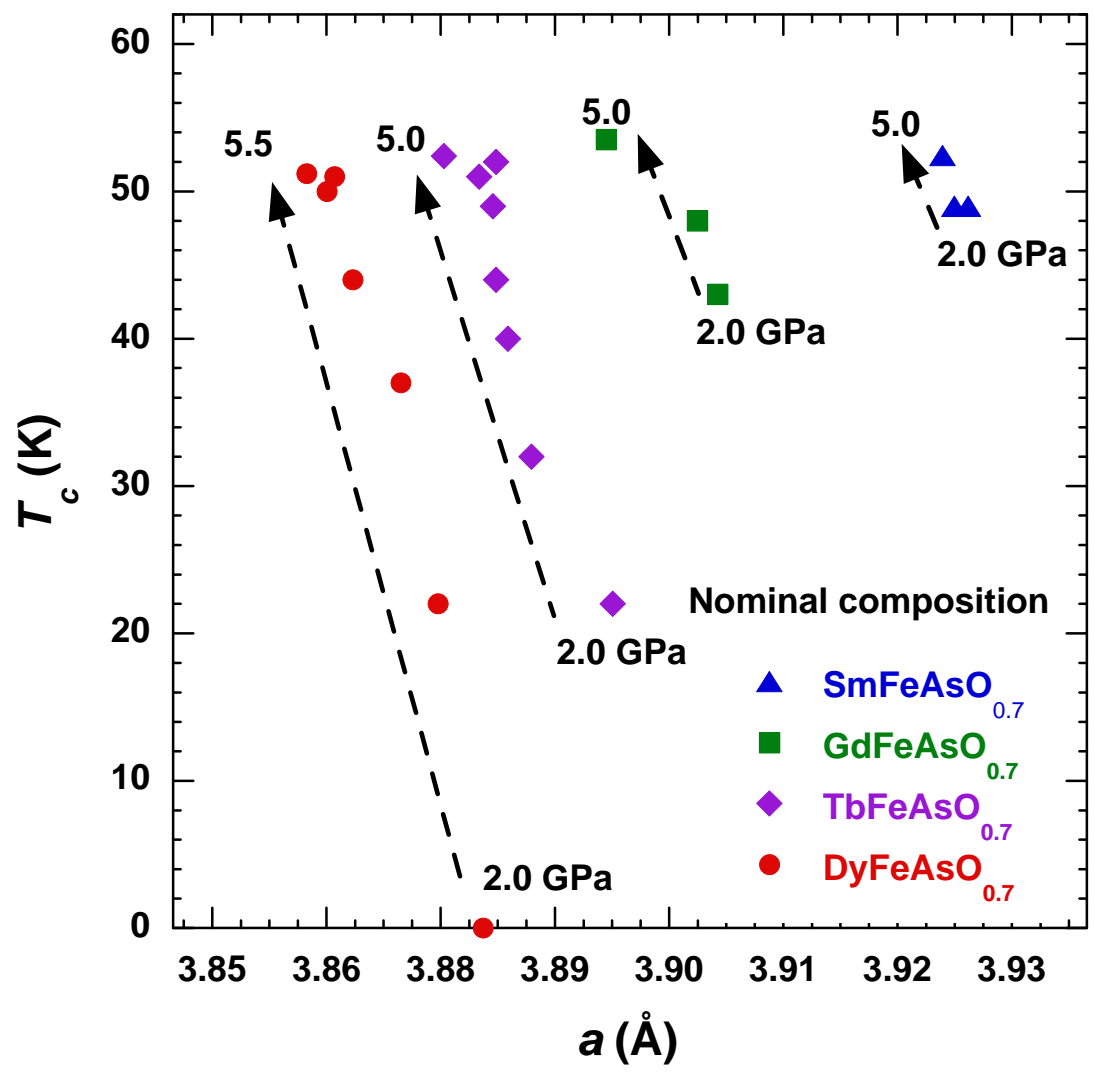

Shirage-Physica C-Figure10 

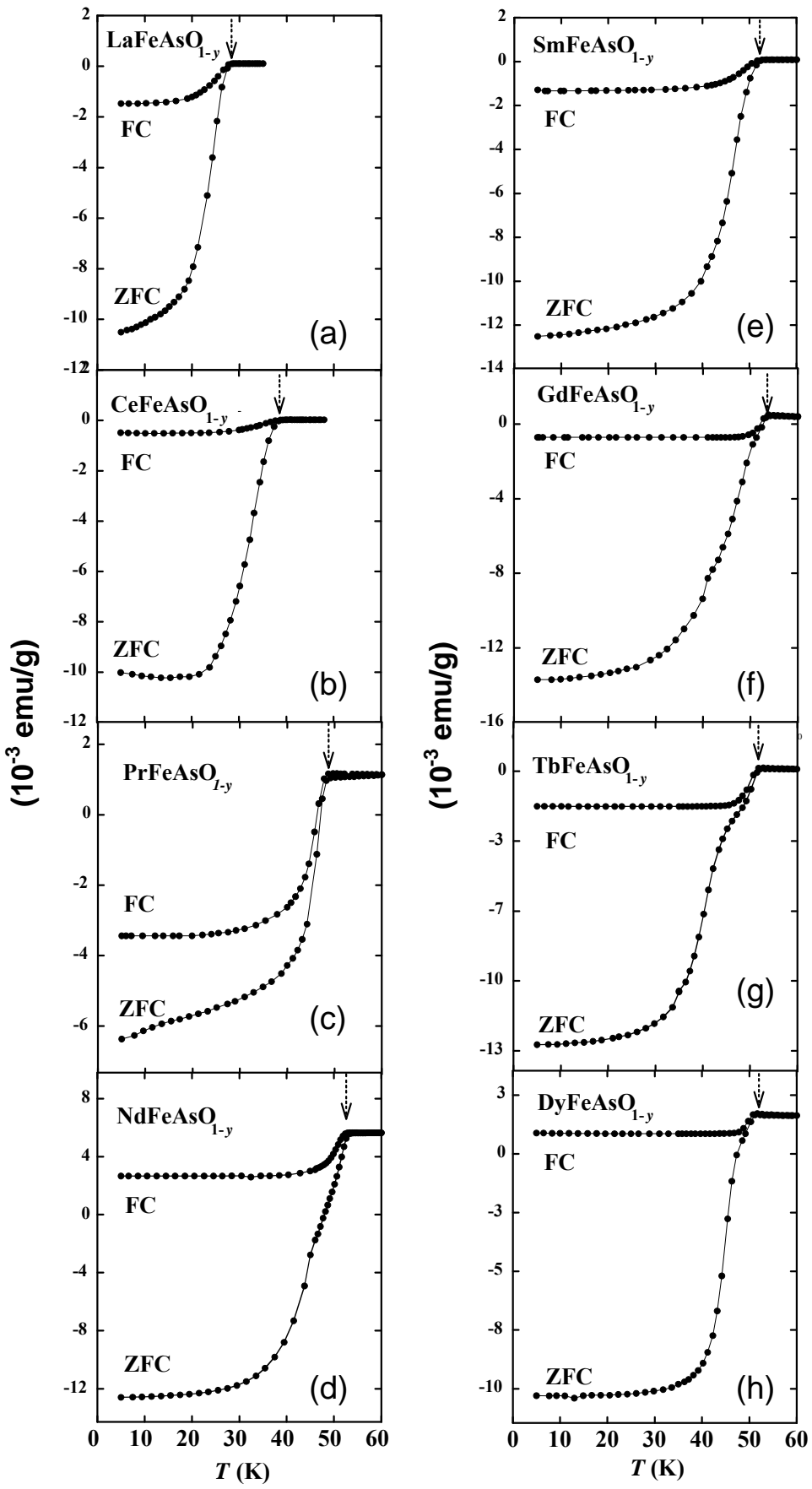

Shirage-Physica C-Figure11 

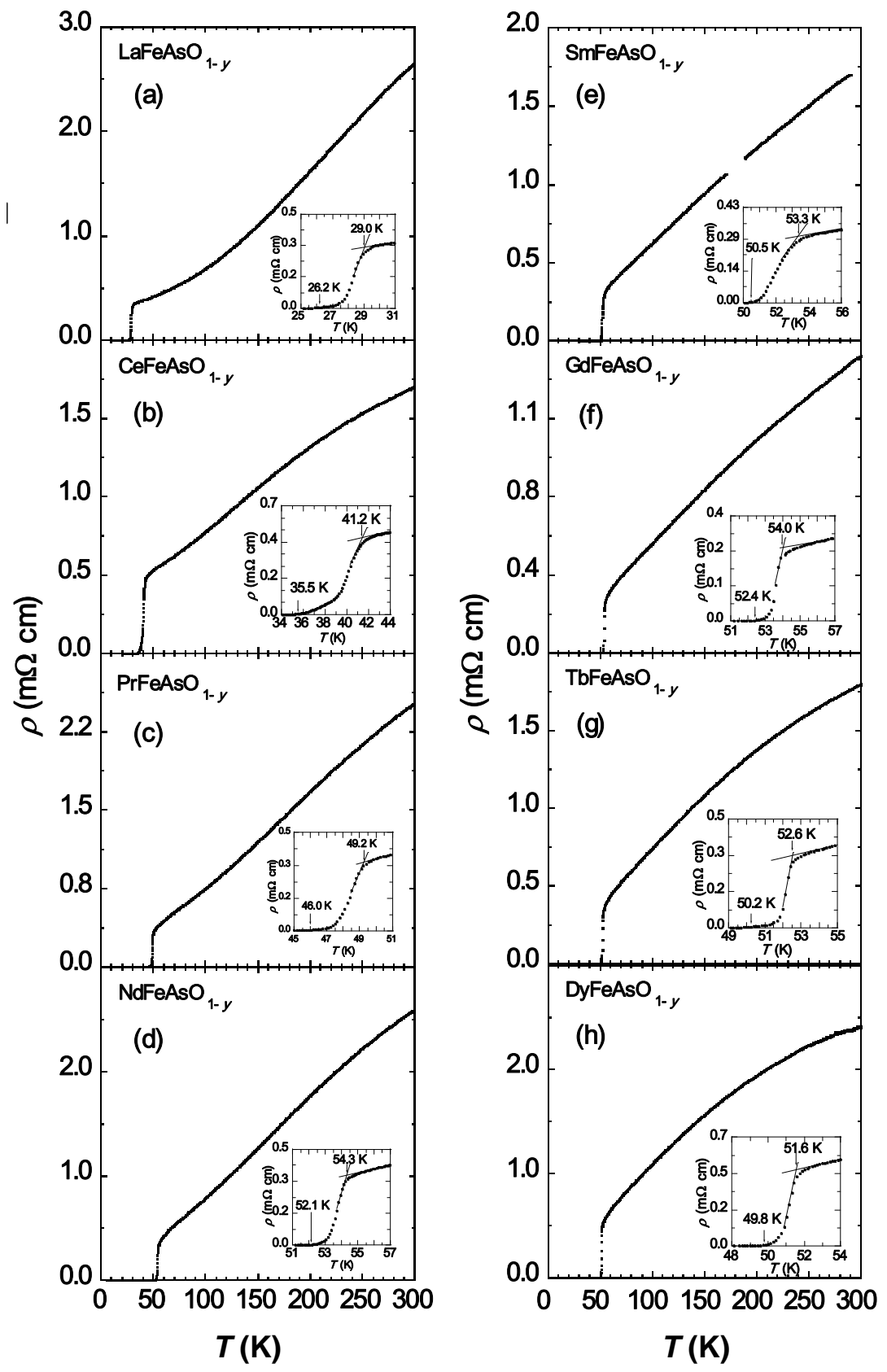

Shirage-Physica C-Figure12 


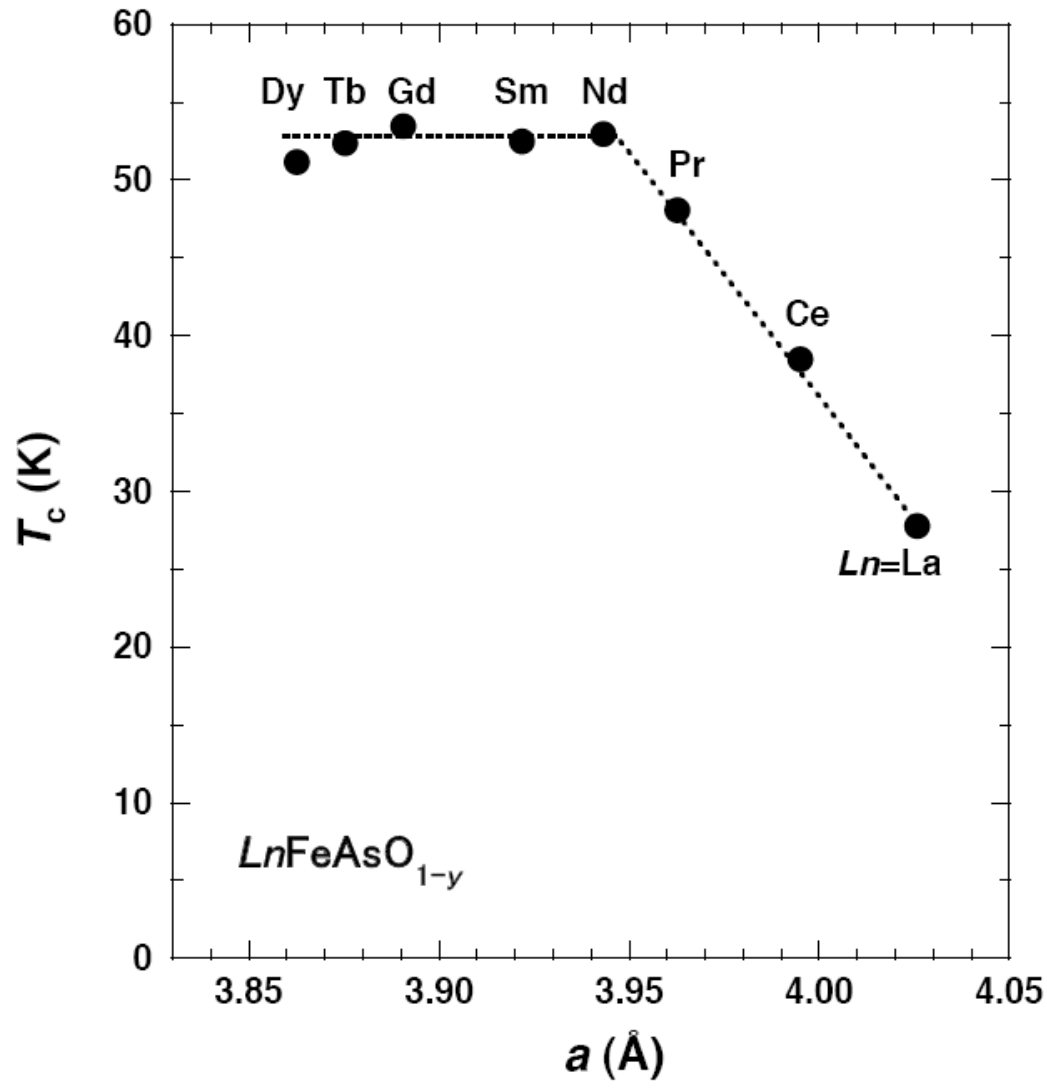

Shirage-Physica C-Figure13 


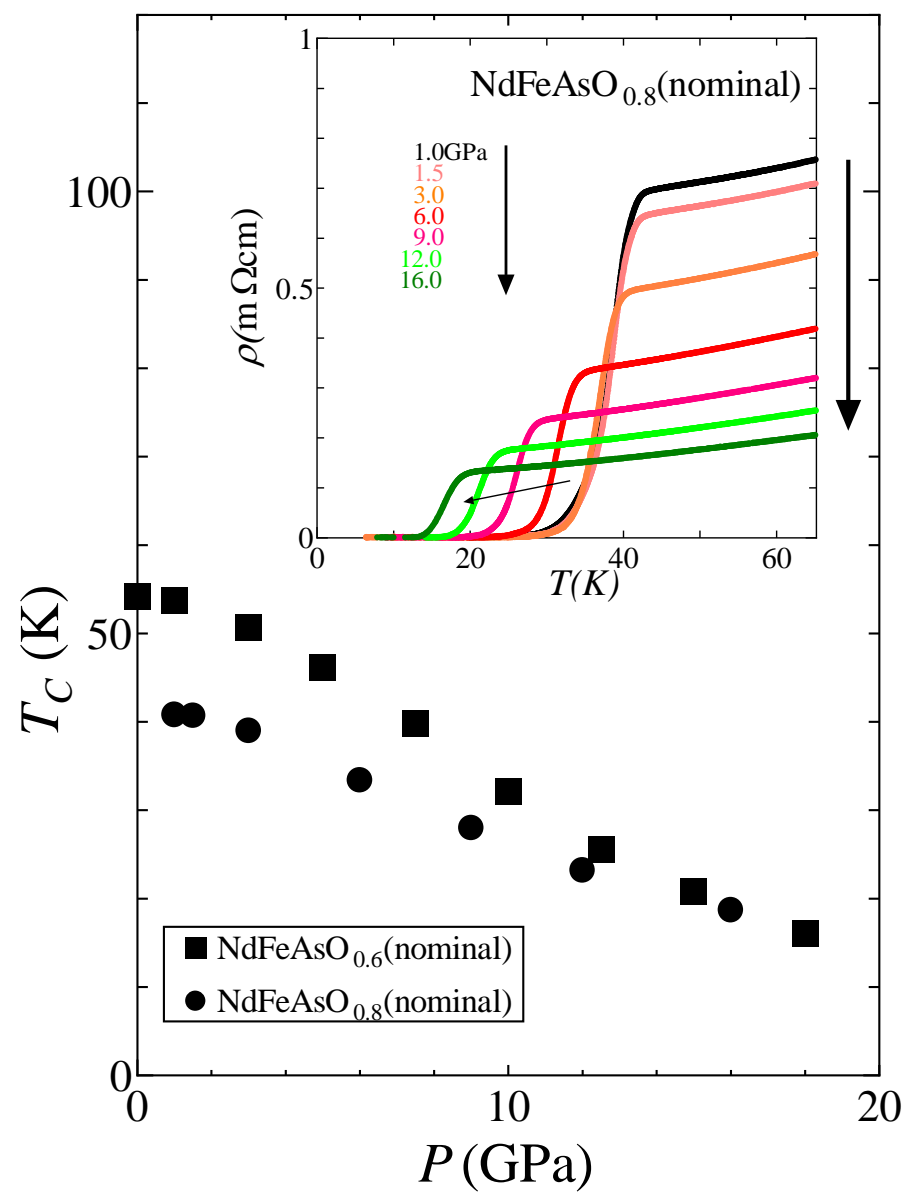

Shirage-Physica C-Figure14 


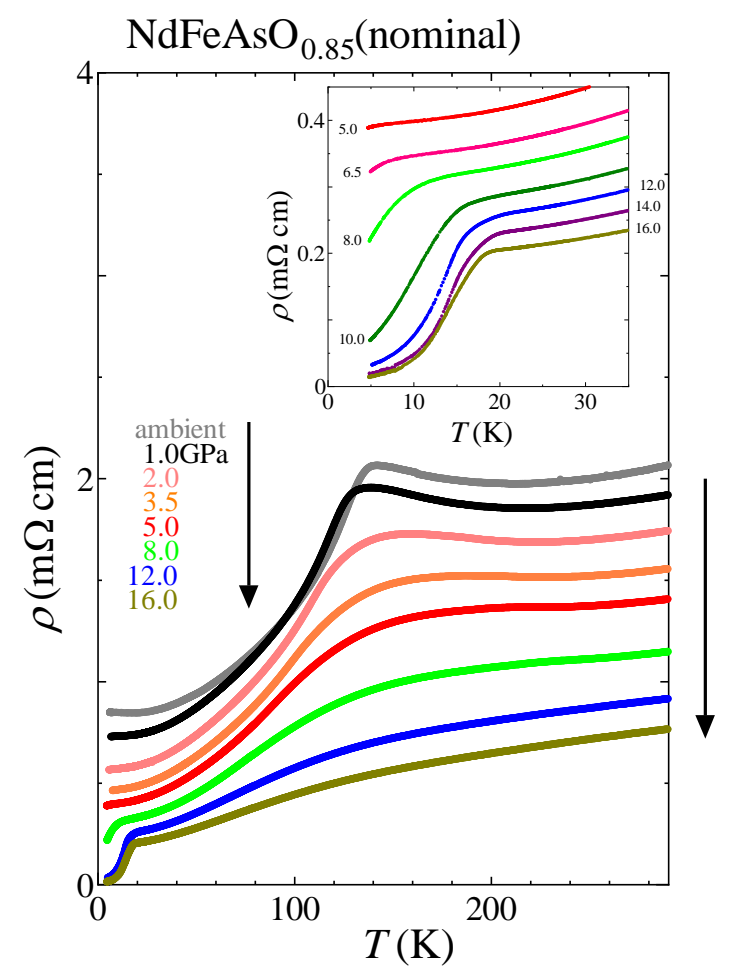

Shirage-Physica C-Figure15 


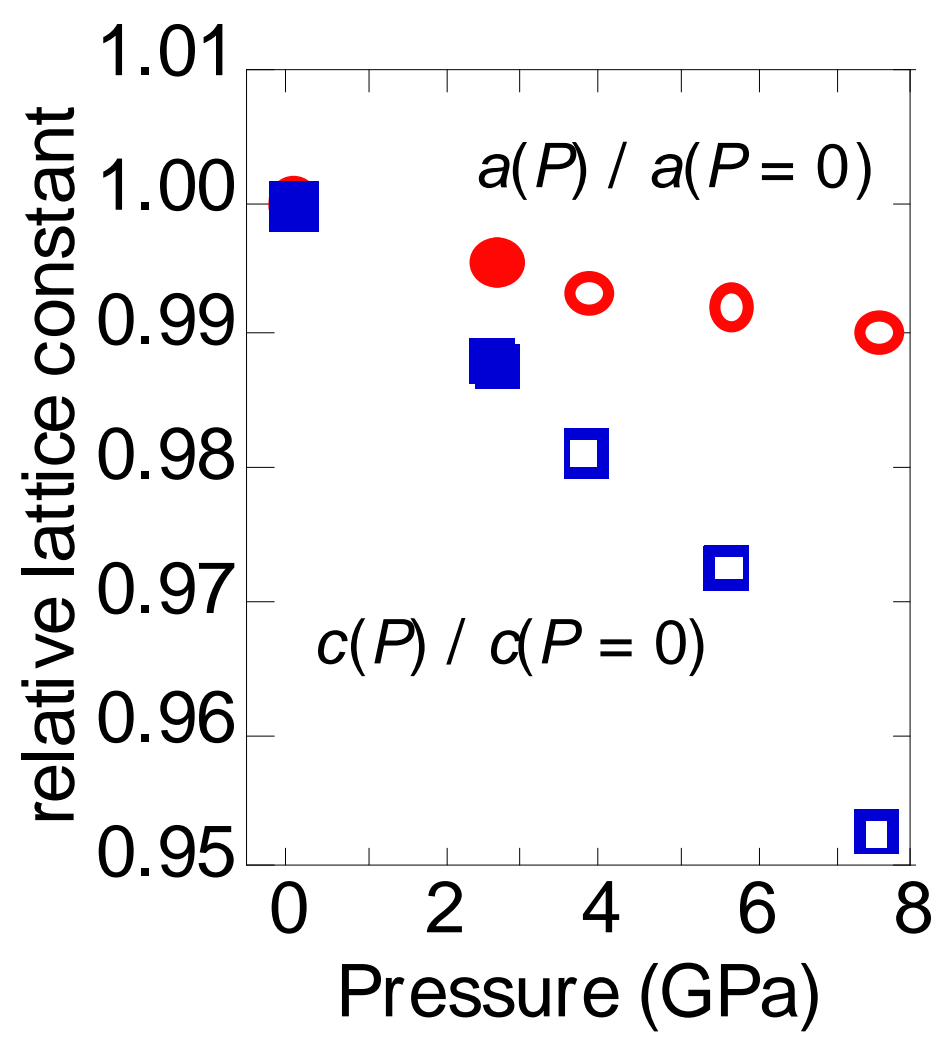

Shirage-Physica C-Figure16 

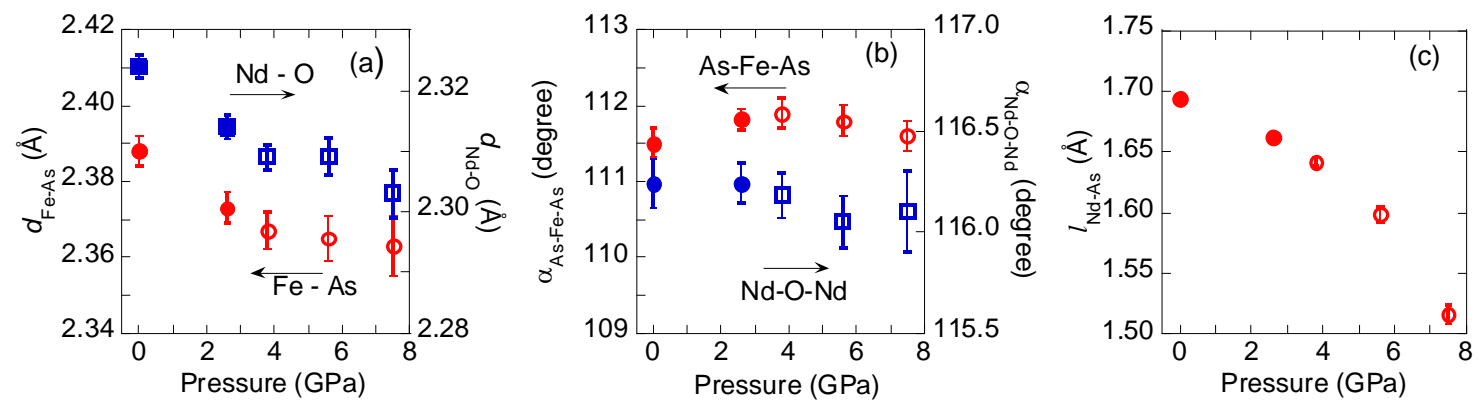

\section{Shirage-Physica C-Figure17}



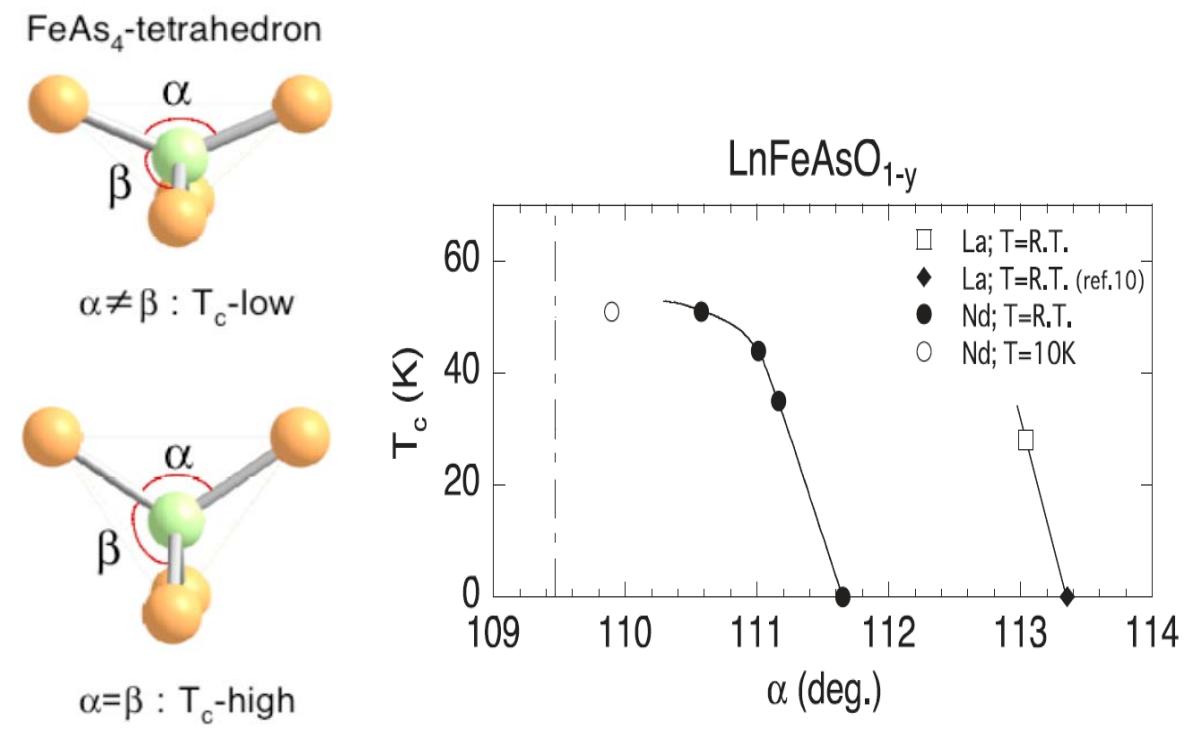

Shirage-Physica C-Figure18 


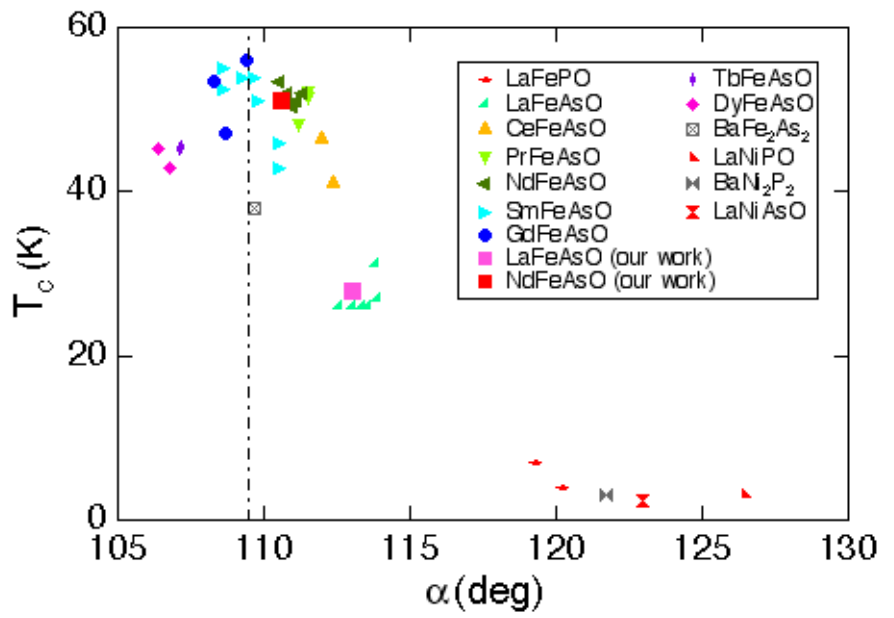

Shirage-Physica C-Figure19 


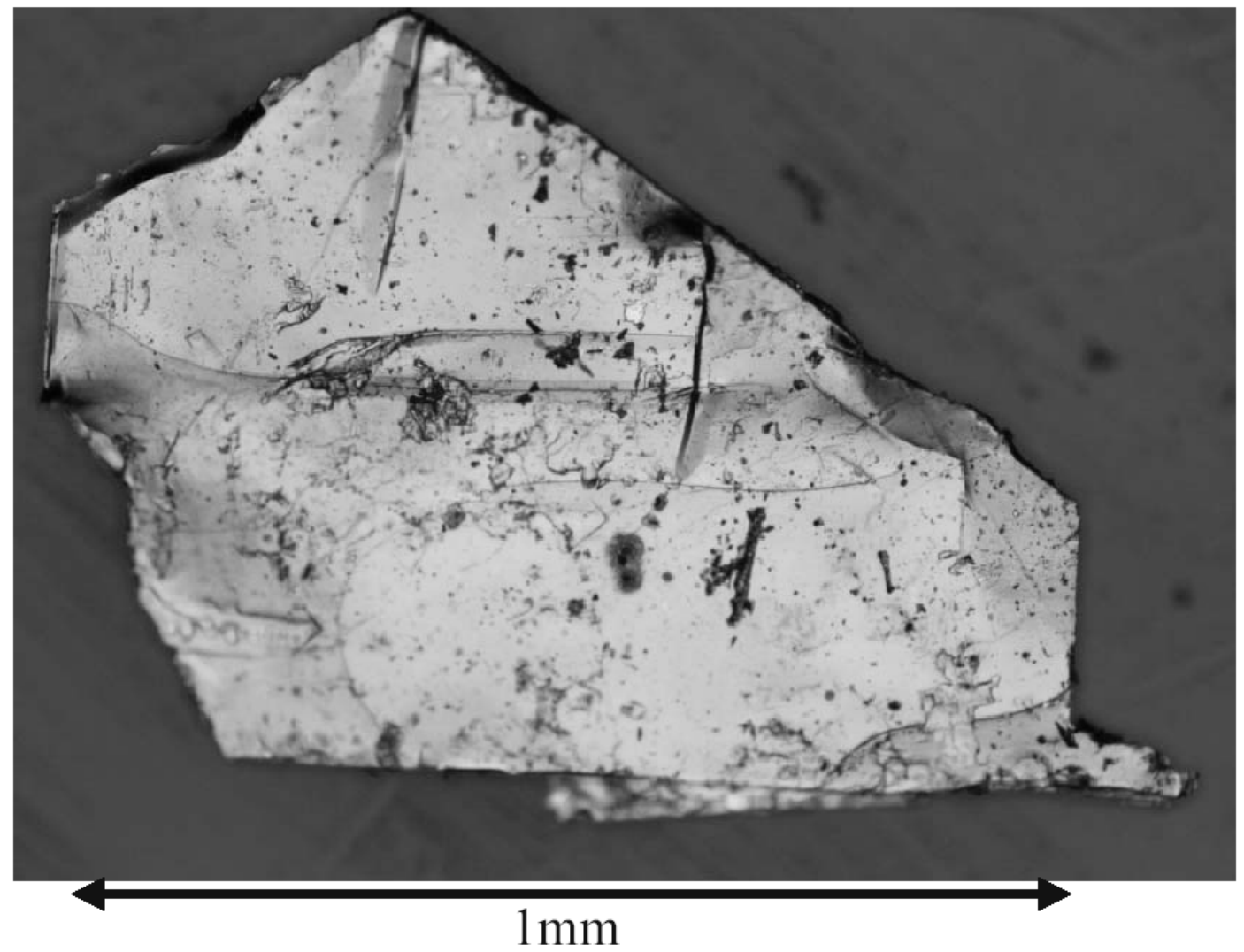

Shirage-Physica C-Figure 20 


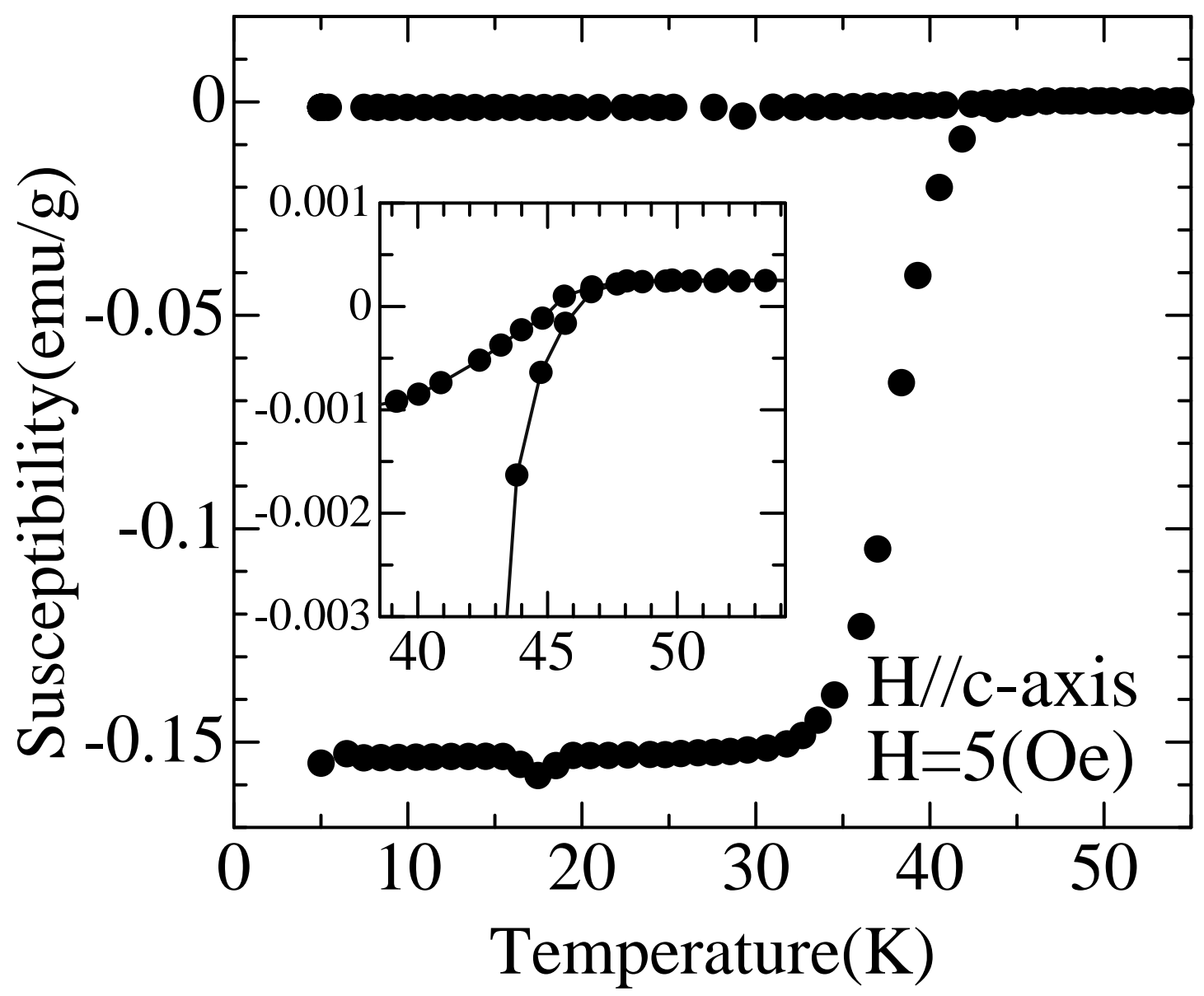

Shirage-Physica C-Figure 21 


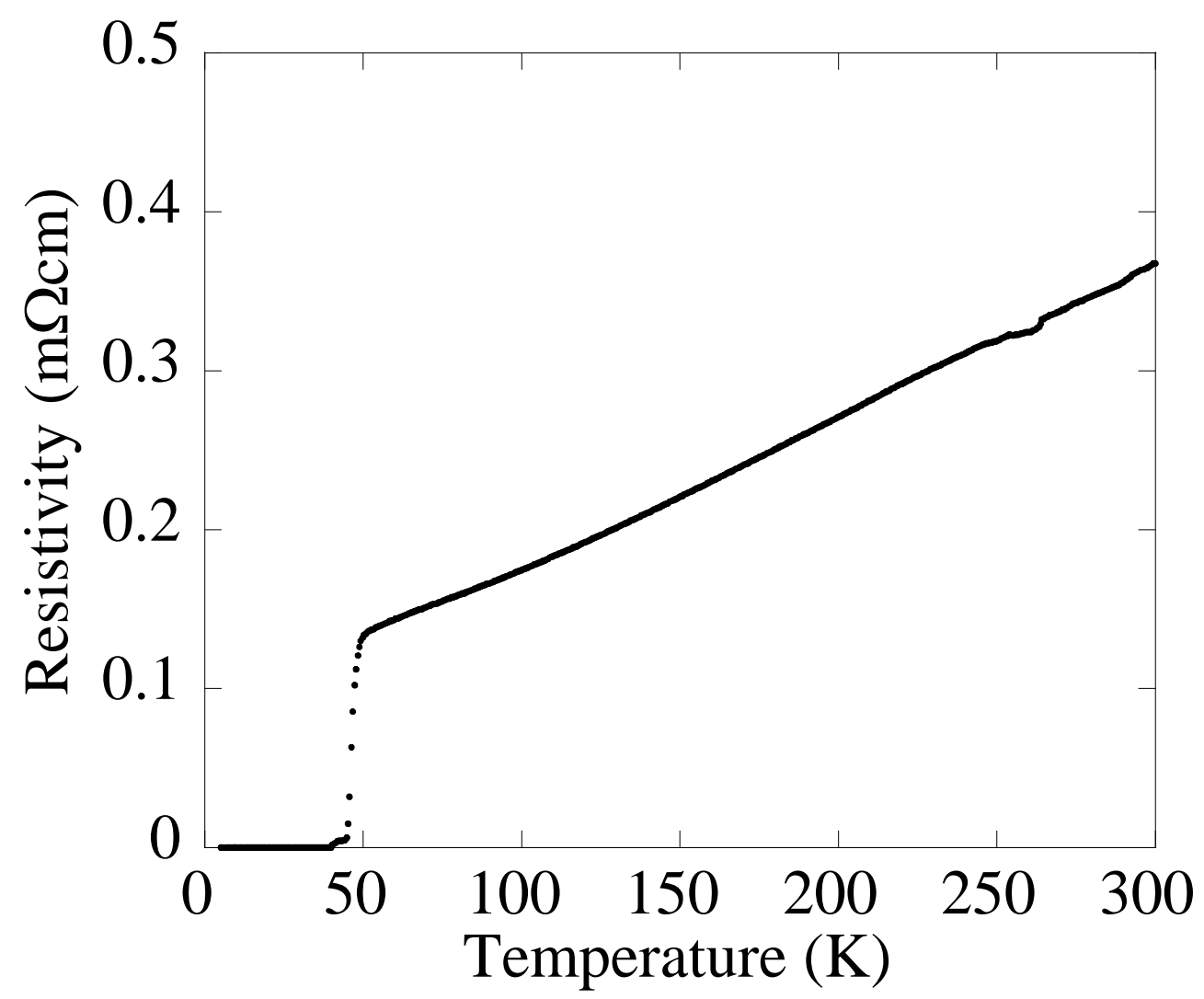

Shirage-Physica C-Figure 22 


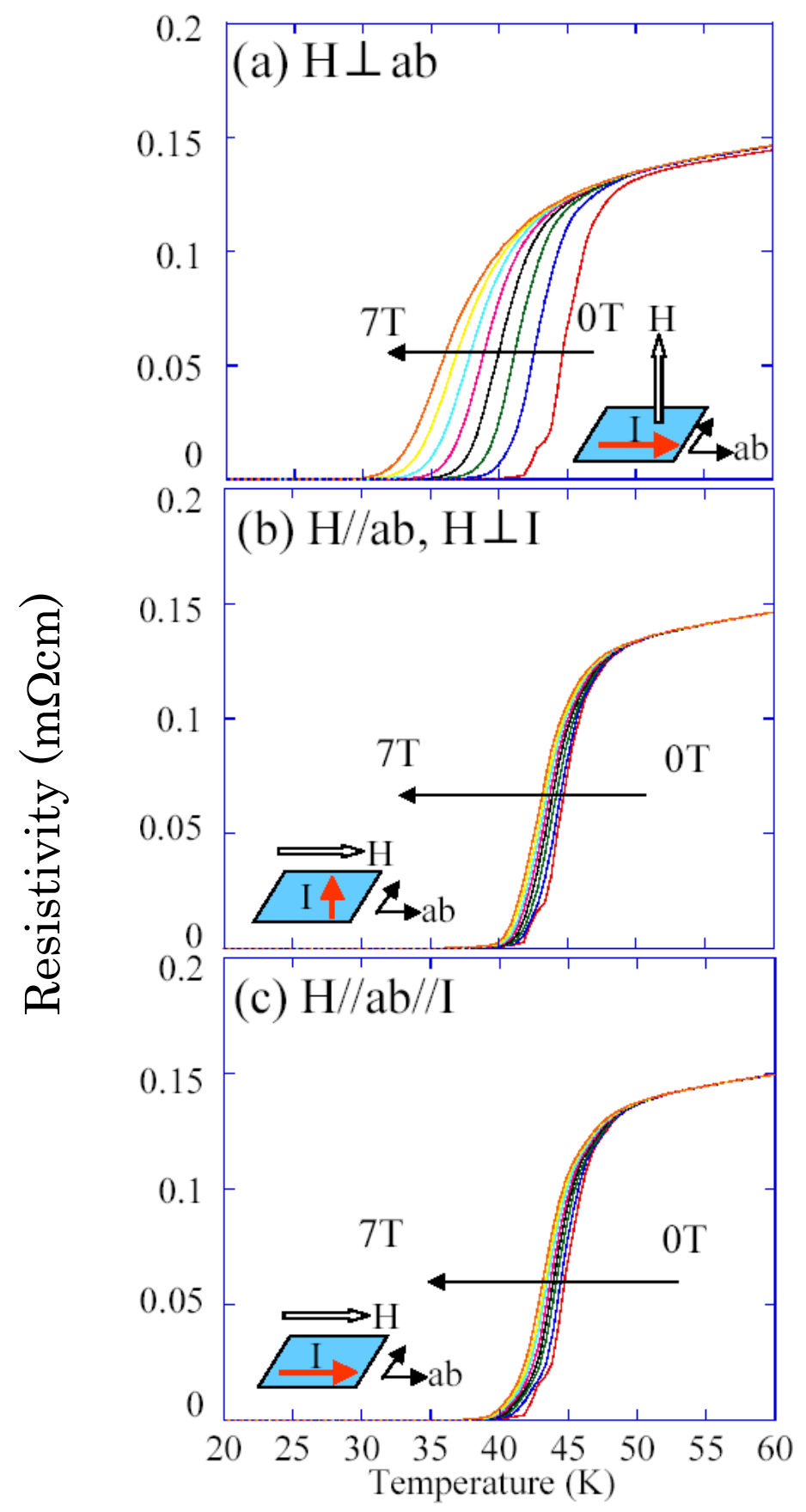

Shirage-Physica C-Figure 23 

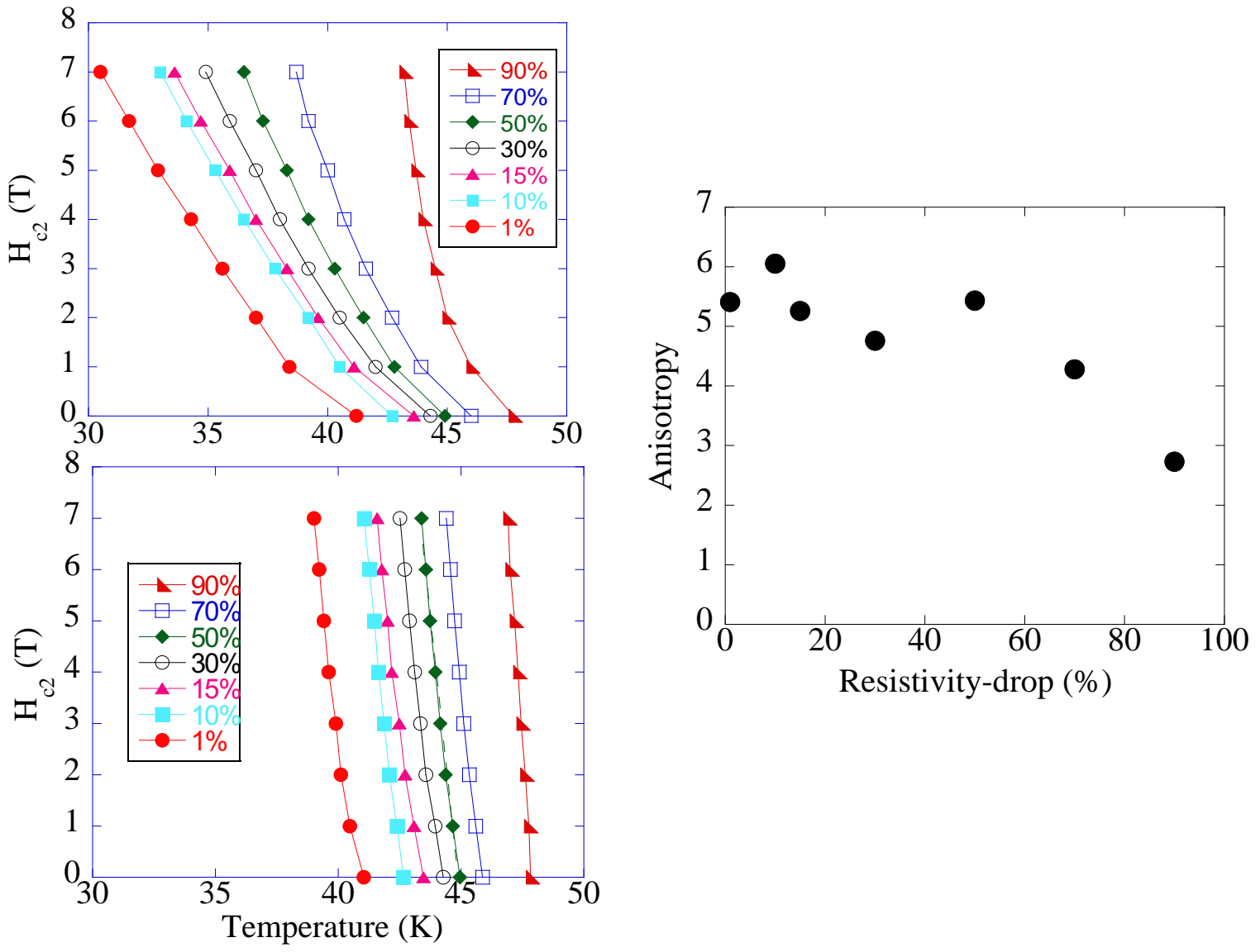

Shirage-Physica C-Figure 24 


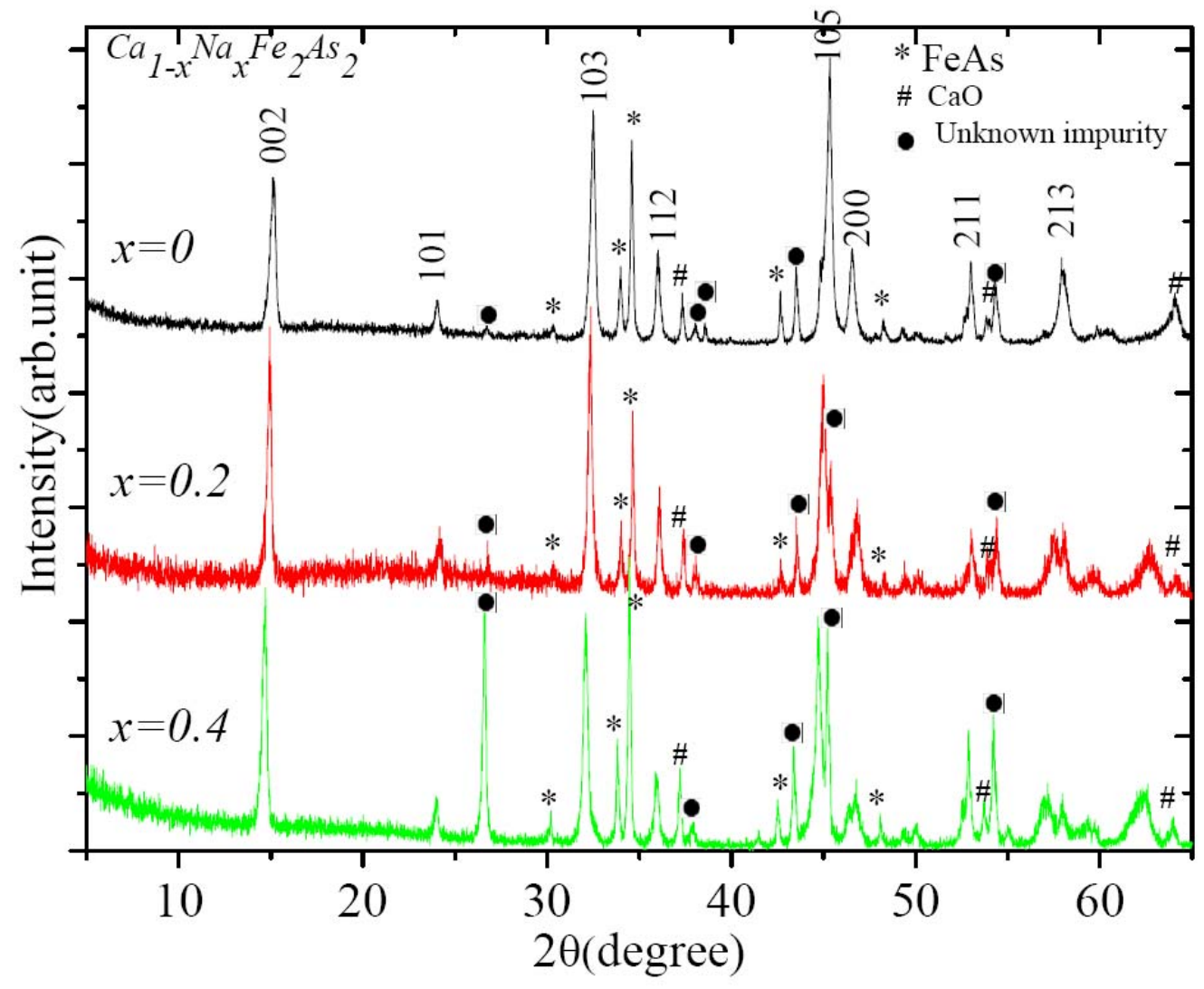

Shirage-Physica C-Figure 25 

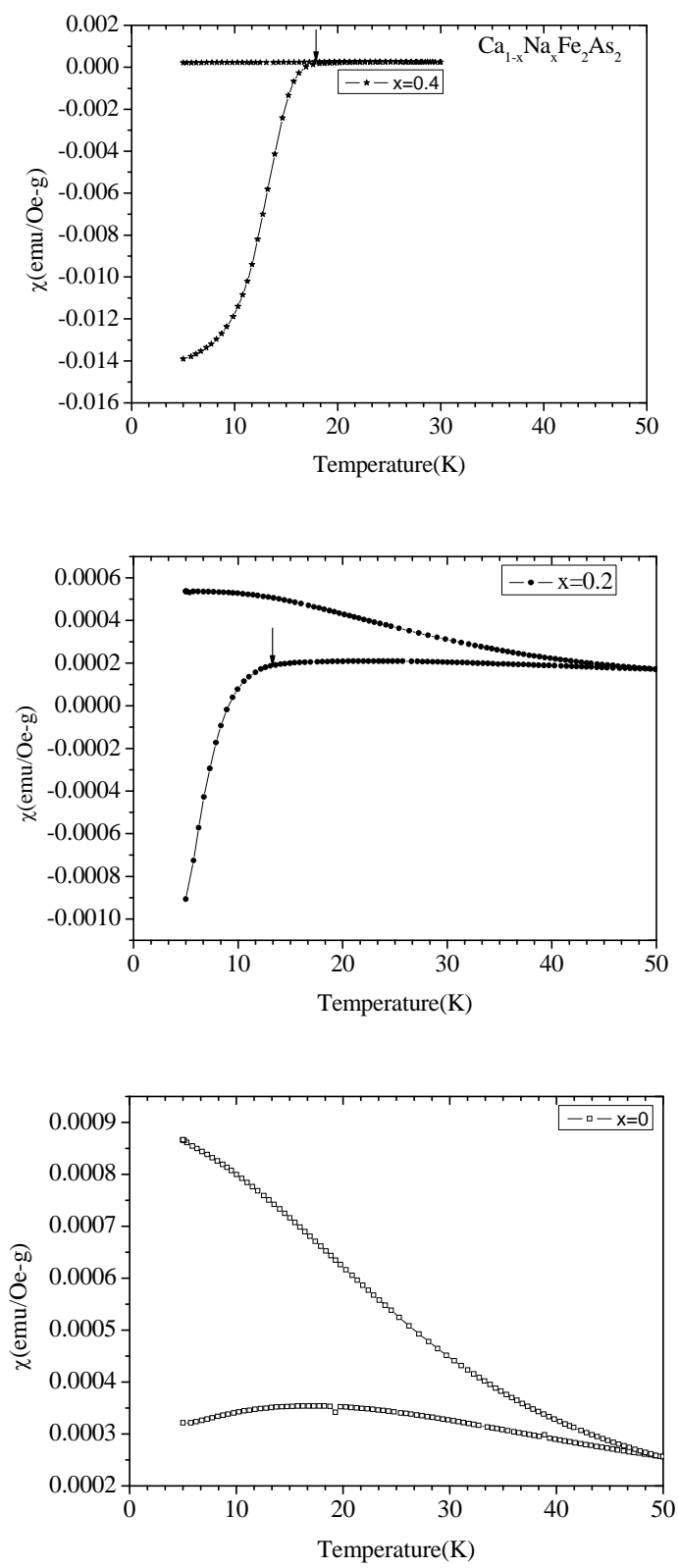

Shirage-Physica C-Figure 26 


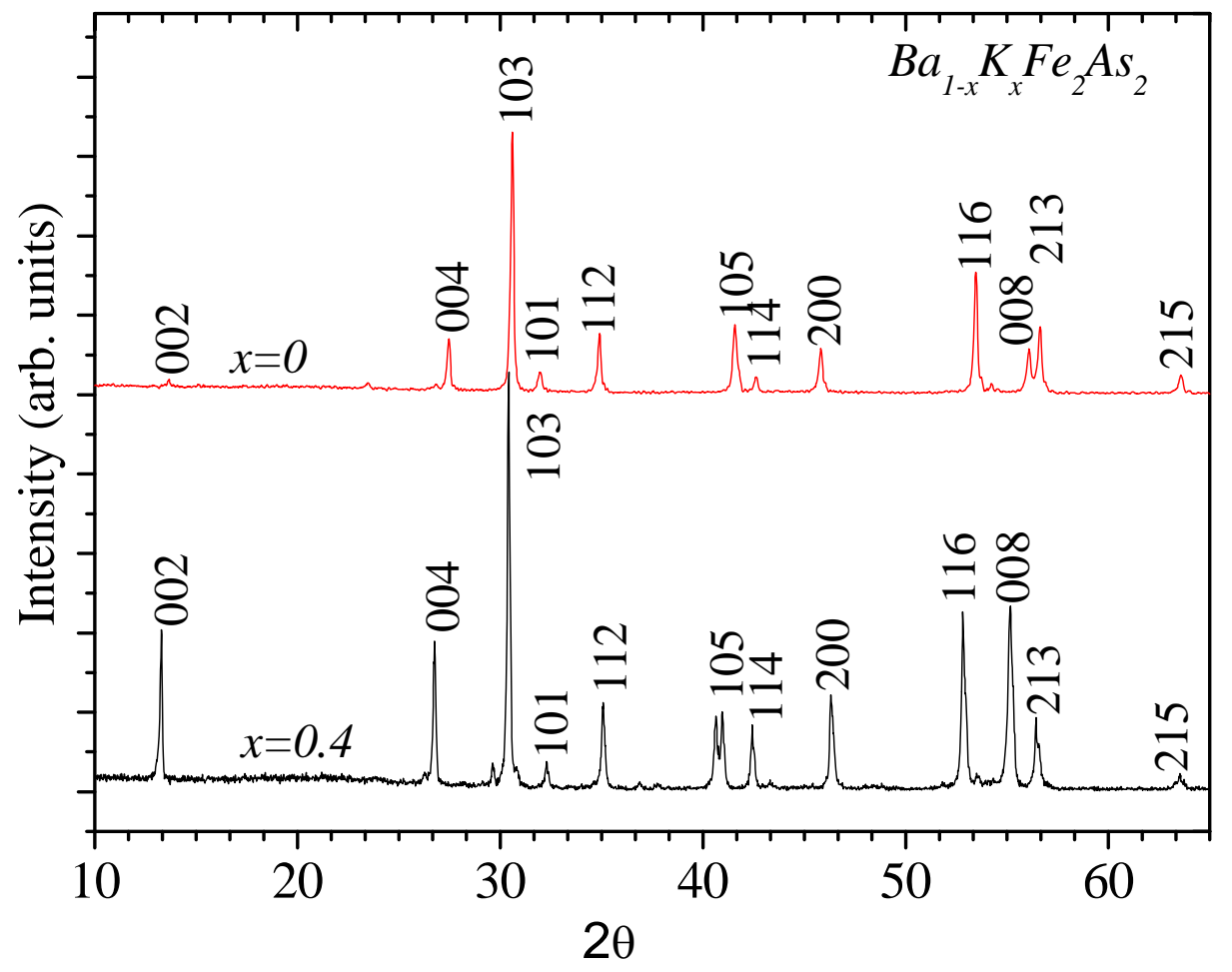

Shirage-Physica C-Figure 27 


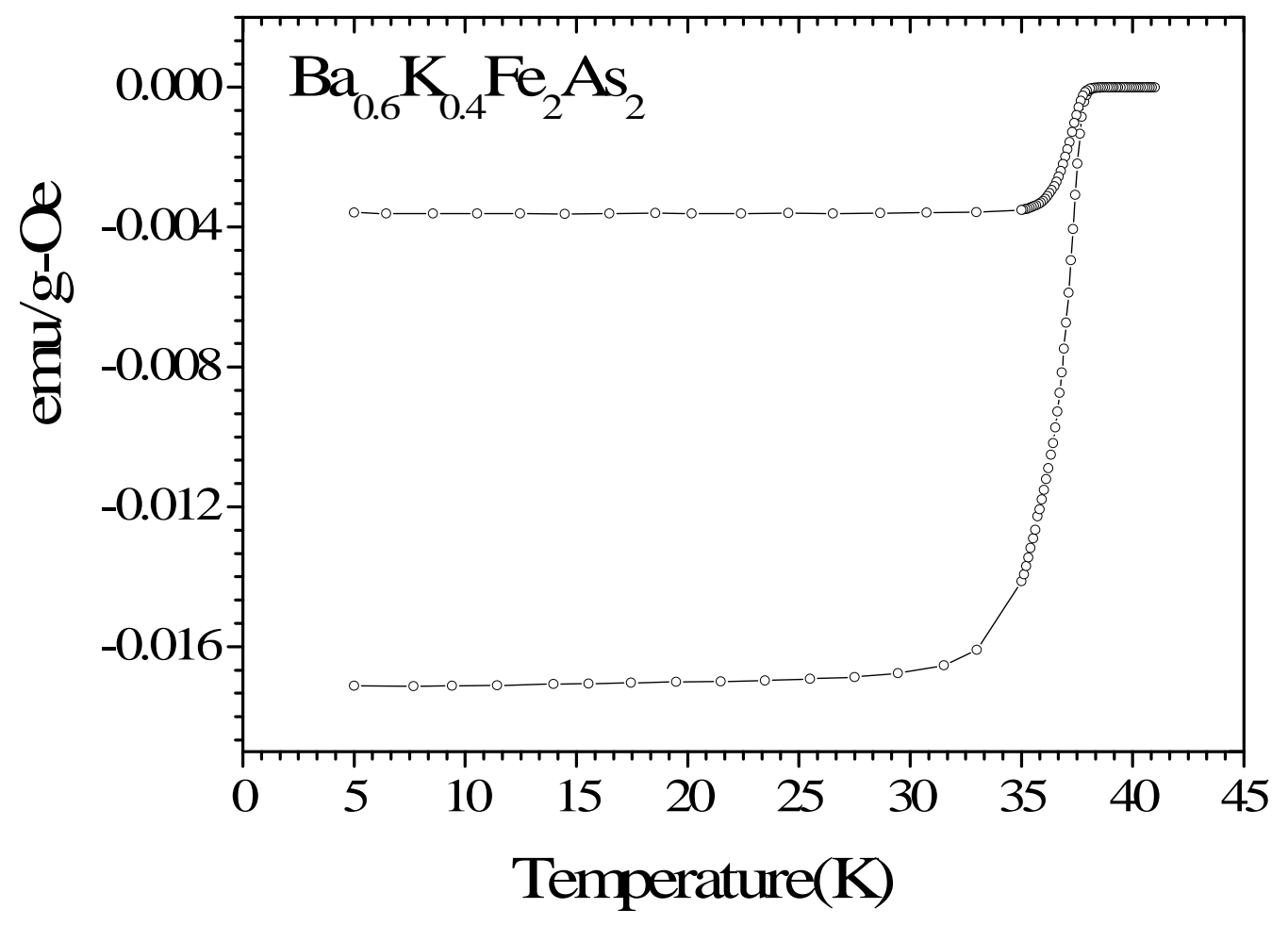

Shirage-Physica C-Figure 28 


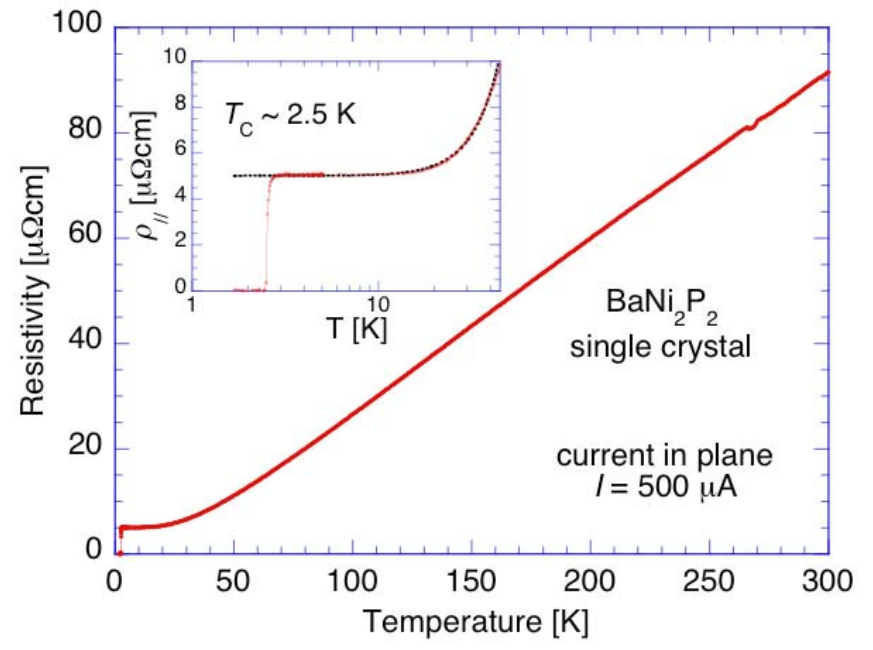

Shirage-Physica C-Figure 29(a) 


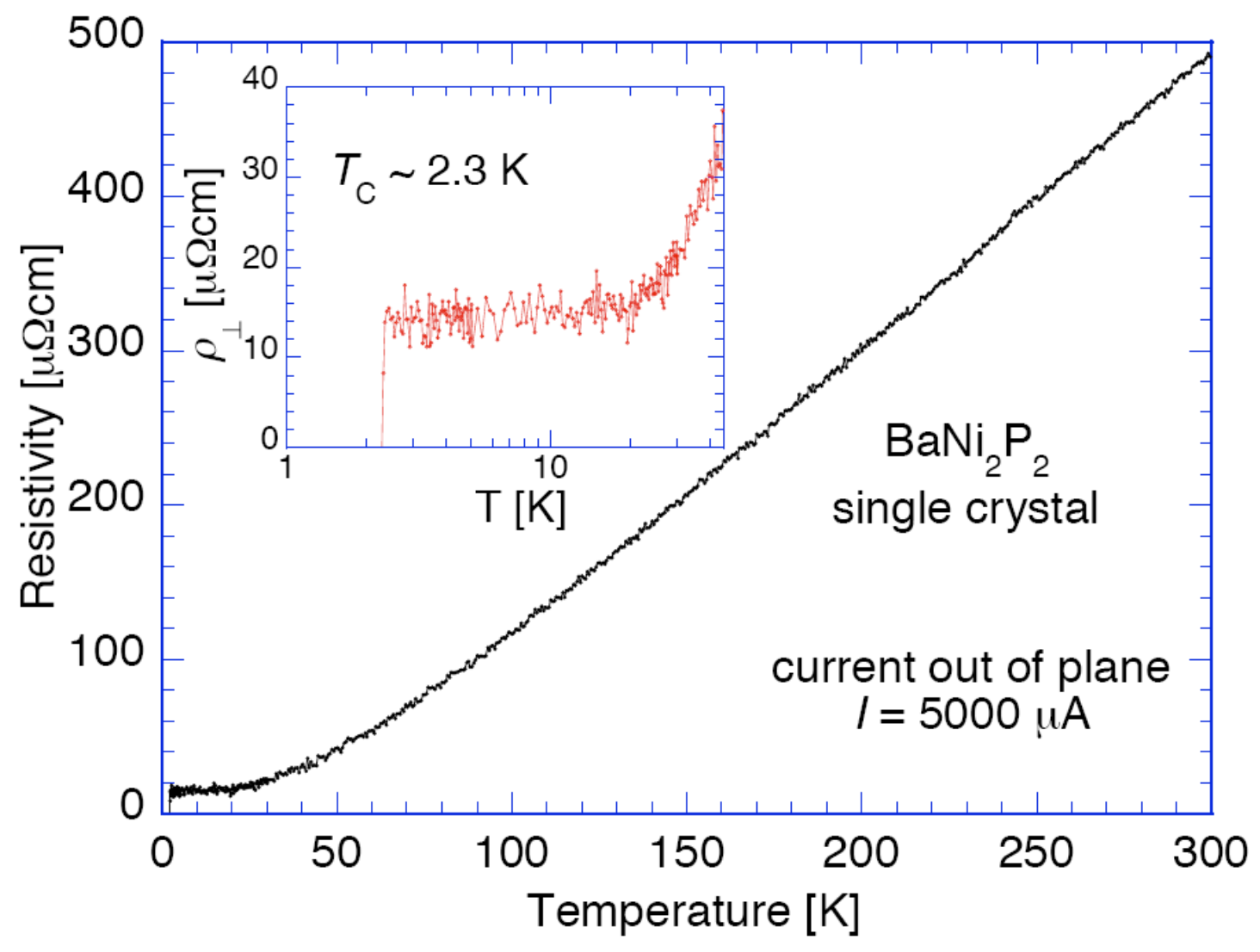

Shirage-Physica C-Figure 29(b) 

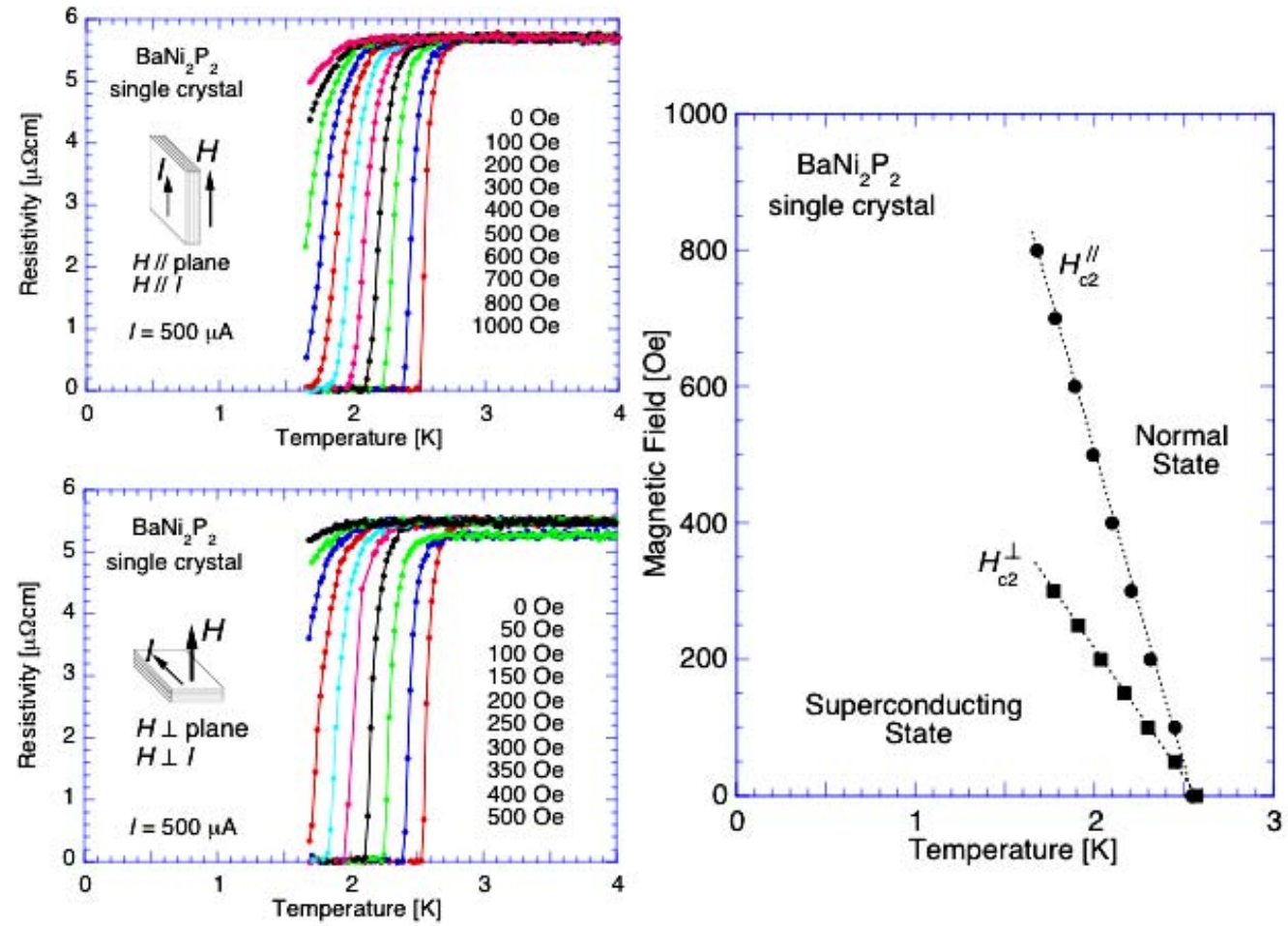

Shirage-Physica C-Figure30 